\title{
Direct numerical simulation of a transitional supercritical binary mixing layer: heptane and nitrogen
}

\author{
By NORA A. OKONG'O AND JOSETTE BELLAN† \\ Jet Propulsion Laboratory, California Institute of Technology, Pasadena, CA 91109-8099, USA
}

(Received 25 September 2000 and in revised form 10 January 2002)

Direct numerical simulations (DNS) of a supercritical temporal mixing layer are conducted for the purpose of exploring the characteristics of high-pressure transitional mixing behaviour. The conservation equations are formulated according to fluctuation-dissipation (FD) theory, which is consistent with non-equilibrium thermodynamics and converges to kinetic theory in the low-pressure limit. According to FD theory, complementing the low-pressure typical transport properties (viscosity, diffusivity and thermal conductivity), the thermal diffusion factor is an additional transport property which may play an increasingly important role with increasing pressure. The Peng-Robinson equation of state with appropriate mixing rules is coupled to the dynamic conservation equations to obtain a closed system. The boundary conditions are periodic in the streamwise and spanwise directions, and of non-reflecting outflow type in the cross-stream direction. Due to the strong density stratification, the layer is considerably more difficult to entrain than equivalent gaseous or droplet-laden layers, and exhibits regions of high density gradient magnitude that become very convoluted at the transitional state. Conditional averages demonstrate that these regions contain predominantly the higher-density, entrained fluid, with small amounts of the lighter, entraining fluid, and that in these regions the mixing is hindered by the thermodynamic properties of the fluids. During the entire evolution of the layer, the dissipation is overwhelmingly due to species mass flux followed by heat flux effects with minimal viscous contribution, and there is a considerable amount of backscatter in the flow. Most of the species mass flux dissipation is due to the molecular diffusion term with significant contributions from the cross-term proportional to molecular and thermal diffusion. These results indicate that turbulence models for supercritical fluids should primarily focus on duplicating the species mass flux rather than the typical momentum flux, which constitutes the governing dissipation in atmospheric mixing layers. Examination of the passive-scalar probability density functions (PDFs) indicates that neither the Gaussian, nor the beta PDFs are able to approximate the evolution of the DNSextracted PDF from its inception through transition. Furthermore, the temperaturespecies PDFs are well correlated, meaning that their joint PDF is not properly approximated by the product of their marginal PDFs; this indicates that the traditional reactive flow modelling based on replacing the joint PDF representing the reaction rate by the product of the marginal PDFs is not appropriate. Finally, the subgrid-scale temperature-species PDFs are also well correlated, and the species PDF exhibits important departures from the Gaussian. These results suggest that classic PDFs used in atmospheric pressure flows would not capture the physics of this supercritical mixing layer, either in an assumed PDF model at the larger scale, or at the subgrid scale.

$\dagger$ Author to whom correspondence should be addressed: Josette.Bellan@jpl.nasa.gov 


\section{Introduction}

Supercritical fluid disintegration is of relevance to many power producing devices operating at high pressure: liquid propulsion rockets, diesel and future gas turbine engines are only a few examples. Some natural phenomena, such as gaseous planet (e.g. Jovian) atmospheres, obey similar physics. Despite the considerable relevance, the understanding of turbulent supercritical fluid disintegration is limited to a few experimental observations. Following the pioneering work of Brown \& Roshko (1974) with density-stratified spatial mixing layers and the further experiments of Atsavapranee \& Gharib (1997) with stratified temporal mixing layers, the more recent Mayer et al. $(1996,1998)$ and Chehroudi, Talley \& Coy (1999) observations of jets focus on fluid disintegration at different pressures. A striking finding of all the most recent experiments is the difference between fluid disintegration at subcritical and supercritical pressures (with respect to the fluid in the jet); Oschwald \& Schik (1999) note that "The thermodynamic state of an injected supercritical fluid is dominant in controlling the jet disintegration. Differences in the injection velocity or momentum flux play a minor role". Whereas atomization of a jet results in the formation of a multitude of ligaments and drops as observed by Chehroudi et al. (1999) under subcritical conditions, past the critical point of the fluid, disintegration assumes the aspect of what Chehroudi et al. (1999) have termed 'fingers', or 'comb-like structures' at transcritical conditions, having an increasingly gaseous appearance with increasing pressure. Raman scattering measurements of the radial density in free nitrogen jets at $4 \mathrm{MPa}$ by Oschwald \& Schik (1999) depicted sharp profiles independent of the injection temperature. This indicates that the occurrence of sharp density gradients may be a distinctive optical feature for some species in environments at supercritical conditions.

In the realm of supercritical jet simulations, Bellan (2000) remarks that there are no models incorporating even minimal information necessary for supercritical fluid description, such as transient effects. This dearth of such jet simulations suggests that it is first appropriate to investigate simpler geometric configurations, such as mixing layers, particularly temporal ones since they benefit from additional computational simplicity. Supercritical temporal mixing layers are density stratified, similarly to those studied by Cortesi, Yadigaroglu \& Banerjee (1998) and Cortesi et al. (1999); however, the former incorporate additional effects associated with real gas equations of state (see Harstad, Miller \& Bellan 1997) and an enlarged transport matrix (see Harstad \& Bellan 2000). This enlarged transport matrix contains, in addition to the familiar, low pressure, diffusion coefficients and thermal conductivity, new transport coefficients, termed the thermal diffusion factors: $\alpha_{T, \alpha \beta}$, where the subscripts $\alpha$ and $\beta$ refer to the species. Harstad \& Bellan (2000) showed that according to the form of the heat flux, one may define two types of thermal diffusion factors, which are related by a thermodynamic function associated with the species. The different role of these two types of $\alpha_{T, \alpha \beta}$ in two-dimensional mixing layers was examined by Miller, Harstad \& Bellan (2001) who also conducted a three-dimensional direct numerical simulation (DNS) of a supercritical temporal mixing layer for a single initial Reynolds number (see definition in $\S 3$ ), $R e_{0}=400$. Even for this mixing layer that did not reach transition, one could detect the distinctive regions of large density gradient magnitude optically observed in experiments (see Miller et al. 2001). Although spatial and temporal mixing layers are inherently different, Metcalfe et al. (1987) remark that they share common optical characteristics. Therefore, the initial optical agreement between observations and the simulation is encouraging. 
The lack of mixing transition in the study of Miller et al. (2001) was scrutinized from an enlarged database by Okong'o \& Bellan (2000) who traced its origin to the competing influences of entrainment, producing strong density gradients (a stabilizing effect), and mixing, reducing the density gradients (a destabilizing effect). These density gradient regions played a considerable role in vorticity production, which was concentrated therein and was predominantly due to the baroclinic torque (Miller et al. 2001). Moreover, these regions also played a pivotal role in the lack of transition through damping of the small scales (Okong'o \& Bellan 2000).

However, as discussed above, most practical interest is in turbulent features of jets and mixing layers. The present study is devoted to the investigation of supercritical mixing layer transition and also of associated special flow features that might not be encountered in gaseous layers. In $\S 2$ of this paper we derive the conservation equations for an arbitrary number of species, based on the validated fluid model of Harstad \& Bellan (2000); these equations reduce to those of Miller et al. (2001) in the case of binary species. The configuration and simulation initial and boundary conditions are explained in $\S 3$, while the numerics is described in $\S 4$. The general mixing layer evolution is discussed in the first subsection of $\S 5$, and the behaviour of crucial variables at transition is illustrated and discussed in the next subsection. Also in $\S 5$, the results are analysed in terms of the irreversible entropy production, i.e. the dissipation, and the contribution of various phenomena to the dissipation is examined. Furthermore, the capability of the 'assumed' probability density functions (PDFs) method is investigated, and the potential of the assumed PDF method for obtaining the filtered density function for use in large-eddy simulation (LES) is explored. The final section is devoted to a summary, conclusions, and a brief discussion of directions for further investigations.

\section{Conservation equations}

The conservation equations originate from the fluctuation-dissipation (FD) theory of Keizer (1987). This theory is consistent with non-equilibrium thermodynamics and converges to kinetic theory in the low pressure limit. It also relates, from first principles, fluxes and forces; this relationship appears only heuristically in continuum theory through an assumed extension of kinetic theory expressions. The FD theory leads to general expressions for the mass and heat fluxes in the evolution equations for the conservative variables; in this section we present both the general form, and the form specific to the heptane-nitrogen mixture under consideration.

\subsection{Dynamic conservation equations}

The compressible form of the conservation equations is

$$
\begin{gathered}
\frac{\partial \rho}{\partial t}+\frac{\partial}{\partial x_{j}}\left[\rho u_{j}\right]=0, \\
\frac{\partial}{\partial t}\left(\rho u_{i}\right)+\frac{\partial}{\partial x_{j}}\left[\rho u_{i} u_{j}+p \delta_{i j}-\tau_{i j}\right]=0, \\
\frac{\partial}{\partial t}\left(\rho e_{t}\right)+\frac{\partial}{\partial x_{j}}\left[\left(\rho e_{t}+p\right) u_{j}-u_{i} \tau_{i j}+q_{I K, j}\right]=0, \\
\frac{\partial}{\partial t}\left(\rho Y_{\alpha}\right)+\frac{\partial}{\partial x_{j}}\left[\rho Y_{\alpha} u_{j}+j_{\alpha j}\right]=0, \quad \alpha \in[1, N],
\end{gathered}
$$


where $t$ denotes the time, $x$ is a Cartesian coordinate, subscripts $i$ and $j$ refer to the spatial coordinates, $u_{i}$ is the velocity, $\rho$ is the density, $e_{t}=e+u_{i} u_{i} / 2$ is the total energy (i.e. internal energy, $e$, plus kinetic energy), $p$ is the thermodynamic pressure, $Y_{\alpha}$ is the mass fraction of species $\alpha$ and $N$ is the number of species. Furthermore, $\tau_{i j}$ is the Newtonian viscous stress tensor

$$
\tau_{i j}=\mu\left[\frac{\partial u_{i}}{\partial x_{j}}+\frac{\partial u_{j}}{\partial x_{i}}-\frac{2}{3} \frac{\partial u_{k}}{\partial x_{k}} \delta_{i j}\right],
$$

where $\delta_{i j}$ is the Kronecker delta function, and $\mu$ is the mixture viscosity which is, in general, a function of the thermodynamic state variables. For an isotropic fluid, $\tau_{i j}$ usually contains terms proportional to both shear and bulk viscosity; however, the FD theory of Keizer (1987) clearly distinguishes between these two contributions: whereas shear viscosity arises from molecular interactions transferring momentum between neighbouring fluid elements, bulk viscosity is related to changes, or different states, of internal energy. Since bulk viscosity effects generally tend to be small (see Bird, Stewart \& Lightfoot 1990), here they are neglected; the value of the bulk viscosity is uncertain or unknown under the best circumstances. In (2.3) $\boldsymbol{q}_{I K}$ denotes the heat flux, where the subscript $I K$ refers to the Irwing-Kirkwood form of the heat flux (see Sarman \& Evans 1992), and in (2.4) $\boldsymbol{j}_{\alpha}$ represents the species mass flux.

The governing equations for the mixing layer comprise (2.1)-(2.4) and the equation of state whose form is discussed in $\S 2.3$. The fluxes $\boldsymbol{j}_{\alpha}$ in (2.4) and $\boldsymbol{q}_{I K}$ in (2.3) are given by the FD theory discussed below.

\subsection{The flux matrix}

The primary result of FD theory is the form of the flux matrix which now contains additional terms with respect to the familiar Fick and Fourier contributions. Following Harstad \& Bellan (2000), the molar flux, $\boldsymbol{J}_{\alpha}$, and $\boldsymbol{q}_{I K}$ can be written as a sum of a set of functions of thermodynamic variables and transport coefficients, each multiplied by a gradient of a thermodynamic variable:

$$
\left.\begin{array}{l}
-\boldsymbol{J}_{\alpha}=\sum_{\beta=1}^{N-1} A_{J, \alpha}^{\beta} \nabla X_{\beta}+B_{J, \alpha} \nabla T+C_{J, \alpha} \nabla p, \\
-\boldsymbol{q}_{I K}=A_{q} \nabla T+\sum_{\beta=1}^{N-1} C_{q}^{\beta} \nabla X_{\beta}+B_{q} \nabla p,
\end{array}\right\}
$$

where the coefficients $A_{J, \alpha}^{\beta}, A_{q}, B_{J, \alpha}, B_{q}, C_{q}^{\beta}$ and $C_{J, \alpha}$ are functions of thermodynamic quantities and transport coefficients, as given by Harstad \& Bellan (2000). Cumbersome mathematical manipulations (see Harstad \& Bellan 2000) lead to the final form of the fluxes as

$$
\boldsymbol{J}_{\alpha}=-n\left[X_{\alpha}\left(D_{T, \alpha} \nabla \ln T+D_{p, \alpha} \nabla \ln p\right)+\sum_{\gamma=1}^{N-1} \mathscr{D}_{\alpha \gamma} \nabla X_{\gamma}\right],
$$

where

$$
D_{T, \alpha} \equiv \sum_{\beta=1}^{N} Y_{\beta}\left[\alpha_{I K, \alpha \beta}-\frac{1}{R_{u} T}\left(m_{\alpha} m_{\beta} / m\right)\left(h_{, \alpha} / m_{\alpha}-h_{, \beta} / m_{\beta}\right)\right] D_{m, \alpha \beta},
$$


DNS of a supercritical mixing layer

$$
\begin{gathered}
D_{p, \alpha} \equiv \frac{1}{R_{u} T} p \sum_{\beta=1}^{N} Y_{\beta}\left(m_{\alpha} m_{\beta} / m\right)\left(v_{, \alpha} / m_{\alpha}-v_{, \beta} / m_{\beta}\right) D_{m, \alpha \beta}, \\
\mathscr{D}_{\alpha \gamma} \equiv \sum_{\beta=1}^{N} D_{m, \alpha \beta}\left(m_{\beta} / m\right)\left(Y_{\beta} \alpha_{D \alpha \gamma}-Y_{\alpha} \alpha_{D \beta \gamma}\right)
\end{gathered}
$$

and

$$
\boldsymbol{q}_{I K}=-\lambda_{I K}^{\prime} \nabla T-n R_{u} T\left(D_{q p} \nabla \ln p+\sum_{\gamma=1}^{N-1} \mathscr{D}_{q \gamma} \nabla X_{\gamma}\right),
$$

where $\lambda_{I K}^{\prime}$ is a form of the thermal conductivity

$$
\begin{gathered}
\lambda_{I K}^{\prime}=\lambda_{I K}-(\rho / T) \sum_{\alpha>\beta=1}^{N} Y_{\alpha} Y_{\beta}\left(h_{, \alpha} / m_{\alpha}-h_{, \beta} / m_{\beta}\right) \alpha_{I K, \alpha \beta} D_{m, \alpha \beta}, \\
D_{q p} \equiv \frac{1}{R_{u} T} m p \sum_{\alpha>\beta=1}^{N} Y_{\alpha} Y_{\beta}\left(v_{, \alpha} / m_{\alpha}-v_{, \beta} / m_{\beta}\right) \alpha_{I K, \alpha \beta} D_{m, \alpha \beta}, \\
\mathscr{D}_{q \gamma} \equiv \sum_{\alpha>\beta=1}^{N}\left(Y_{\beta} \alpha_{D \alpha \gamma}-Y_{\alpha} \alpha_{D \beta \gamma}\right) \alpha_{I K, \alpha \beta} D_{m, \alpha \beta} .
\end{gathered}
$$

In our notation, $X_{\alpha}$ represents the molar fraction, $T$ is the temperature, $m_{\alpha}$ is the molar mass, while $m$ is the average molar mass of the mixture calculated from the individual species molar masses, $m=\sum_{\gamma=1}^{N} m_{\gamma} X_{\gamma} ; n=\rho / m$ is the molar density; $v_{, \alpha}=$ $\left(\partial v / \partial X_{\alpha}\right)_{T, p, X_{\beta}(\beta \neq \alpha)}$ is the partial molar volume, where the molar volume is $v=1 / n$; $h_{, \alpha}=\left(\partial h / \partial X_{\alpha}\right)_{T, p, X_{\beta}(\beta \neq \alpha)}$ is the partial molar enthalpy, where the molar enthalpy is $h=G-T(\partial G / \partial T)_{p, X}$ with $G$ being the Gibbs energy; $R_{u}$ is the universal gas constant; $D_{m, \alpha \beta}$ is the (symmetric) diffusion coefficient matrix; $\alpha_{I K, \alpha \beta}$ is the (antisymmetric) thermal diffusion coefficient matrix, representing the new set of transport coefficients potentially important at high pressures; $\lambda$ is the thermal conductivity, as $\lim _{p \rightarrow 0} \lambda=$ $\lambda_{K T}$ (see Chapman \& Cowling 1970) where the subscript $K T$ denotes the kinetic theory value; and the mass and molar fractions are related by $Y_{\alpha}=X_{\alpha} m_{\alpha} / m$. The mass diffusion factors, $\alpha_{D \alpha \beta}$, are calculated from thermodynamics as

$$
\alpha_{D \alpha \beta}=\partial X_{\alpha} / \partial X_{\beta}+X_{\alpha} \partial \ln \gamma_{\alpha} / \partial X_{\beta},
$$

with $\gamma_{\alpha} \equiv \varphi_{\alpha} / \varphi_{\alpha}^{o}$, where $\varphi$ is the fugacity coefficient and the superscript $o$ denotes the pure $\left(X_{\alpha}=1\right)$ limit.

For a binary mixture, such as the one considered here, we duplicate the form of the fluxes given in Miller et al. (2001) by noting that $\boldsymbol{j}_{\alpha}=m_{\alpha} \boldsymbol{J}_{\alpha}$ and having $\alpha$ and $\beta$ take the values $h$ (heptane) and $n$ (nitrogen):

$$
\begin{gathered}
q_{I K, j}=-\left[\lambda_{I K}^{\prime} \frac{\partial T}{\partial x_{j}}+\alpha_{I K} R_{u} T\left(\frac{m}{m_{n} m_{h}}\right) j_{h j}^{\prime}\right], \\
j_{h j}=-\left[j_{h j}^{\prime}+\alpha_{B K} Y_{n} Y_{h} \frac{\rho D}{T} \frac{\partial T}{\partial x_{j}}\right], \\
j_{h j}^{\prime}=\rho D\left[\alpha_{D} \frac{\partial Y_{h}}{\partial x_{j}}+\frac{Y_{n} Y_{h}}{R_{u} T}\left(\frac{m_{n} m_{h}}{m}\right)\left(\frac{v_{, h}}{m_{h}}-\frac{v_{, n}}{m_{n}}\right) \frac{\partial p}{\partial x_{j}}\right],
\end{gathered}
$$


where $\alpha_{B K}$ is the thermal diffusion factor associated with the Bearman-Kirkwood form of the heat flux (see Sarman \& Evans 1992). For binary species, there is a single value of the diffusion coefficient $D_{m, \alpha \beta}$ so that $D=D_{h n}=D_{n h} ; D_{h h}$ and $D_{n n}$ do not appear in the equations. In the same manner, there is only a single value for the thermal diffusion factors, $\alpha_{I K, \alpha \beta}$, and $\alpha_{I K}=\alpha_{I K, h n}=-\alpha_{I K, n h}$, with $\alpha_{I K, h h}=\alpha_{I K, n n}=0$. According to Harstad \& Bellan (2000)

$$
\alpha_{I K}=\alpha_{B K}+\frac{1}{R_{u} T}\left(\frac{m_{n} m_{h}}{m}\right)\left(\frac{h_{, h}}{m_{h}}-\frac{h_{, n}}{m_{n}}\right),
$$

and it is thus necessary to specify only one form of the thermal diffusion factors, the two being related by a thermodynamic function. Also, there is a single value of the mass diffusion factor, $\alpha_{D \alpha \beta}$, and according to the Gibbs-Duhem relationship, $\alpha_{D}=\alpha_{D, h h}=\alpha_{D, n n}=-\alpha_{D, h n}=-\alpha_{D, n h}$.

\subsection{Equations of state}

Following Miller et al. (2001), we represent the binary mixture by the Peng-Robinson (PR) equation of state (EOS) because it is computationally one of the simplest, and because the pure species reference states were found to be accurate to better than $1 \%$ relative error through comparisons with the accurate state equations of Harstad et al. (1997) over the range of variables used in this study. The cubic PR EOS is

$$
p=R_{u} T /\left(v-B_{m}\right)-A_{m} /\left(v^{2}+2 v B_{m}-B_{m}^{2}\right),
$$

where $A_{m}$ and $B_{m}$ are functions of composition and temperature (see Harstad et al. 1997 and Miller et al. 2001).

The properties of present interest are $\alpha_{D}, h$, the constant pressure molar heat capacity $C_{p}=(\partial h / \partial T)_{p, X}$, and the speed of sound $a_{s}=\sqrt{1 / \rho \kappa_{s}}$, all calculated in a self-consistent manner from the same EOS, where the isentropic compressibility, $\kappa_{s}$, is related to the expansivity, $\alpha_{v}$, and the isothermal compressibility, $\kappa_{T}$, through

$$
\kappa_{s}=\kappa_{T}-v T \alpha_{v}^{2} / C_{p},
$$

and

$$
\kappa_{T}=\frac{-1}{v(\partial p / \partial v)_{T, X}}, \quad \alpha_{v}=-\frac{(\partial p / \partial T)_{v, X}}{v(\partial p / \partial v)_{T, X}} .
$$

These equations specify the entire thermodynamics of the binary mixture. The detailed thermodynamic expressions are presented in Miller et al. (2001).

\subsection{Transport coefficients}

To close the formulation, one needs to specify the transport coefficients $\mu, D, \lambda$, or alternately the Reynolds number, $R e$, the Schmidt number, $S c$, the Prandtl number, $P r$, and $\alpha_{I K}\left(\right.$ or $\left.\alpha_{B K}\right)$.

The value of the thermal diffusion factor adopted is $\alpha_{I K}=0.1$, determined by Harstad \& Bellan (2000) first from a model calibration through comparisons with microgravity experiments, and further validated in the same study by comparing with other microgravity data.

Based on accurate species transport properties calculated as in Harstad \& Bellan (1998), contour plots (not shown) of $S c, P r$ and the viscosity were constructed. The 


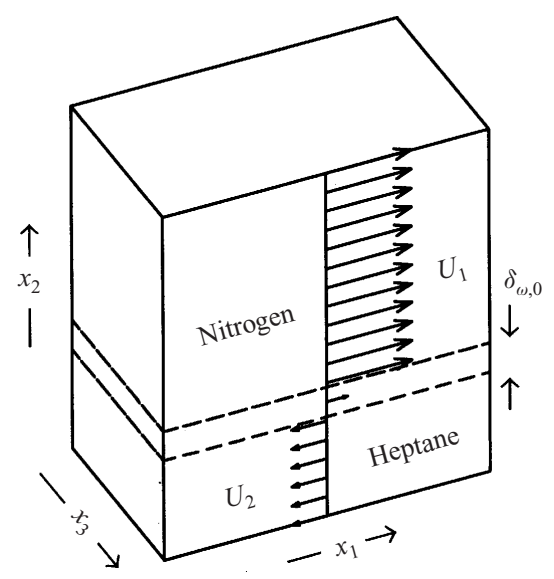

FIGURE 1. Configuration of the heptane-nitrogen mixing layer.

following correlations of these contour plots:

$$
\begin{gathered}
S c=\frac{\mu}{\rho \alpha_{D} D}=1.5-Y_{h}, \quad \operatorname{Pr}=\frac{\mu C_{p} / m}{\lambda}=\frac{S c}{2 \exp \left(-3 Y_{h} / 2\right)}, \\
\mu=\mu_{R}\left(\frac{T}{\left(T_{1}+T_{2}\right) / 2}\right)^{0.7}(T \text { in Kelvins }),
\end{gathered}
$$

where $\mu_{R}$ is a reference viscosity and the reference temperatures $T_{1}$ and $T_{2}$ correspond to the free-stream temperatures for mixing layer simulations, rendered an accurate quantitative representation of these quantities. Equations (2.23)-(2.24) hold in the following range of thermodynamic state space: $500 \mathrm{~K} \leqslant T \leqslant 1100 \mathrm{~K}, 40 \mathrm{~atm} \leqslant p \leqslant$ $80 \mathrm{~atm}$, and $0 \leqslant Y_{h} \leqslant 1$. This particular region is chosen because it is of interest both for diesel and gas turbine engine studies.

The value of the reference viscosity is determined by the specified value of the initial Reynolds number (see below).

\section{Configuration and boundary conditions}

The temporally developing mixing layer configuration is depicted in figure 1 , which shows the definition of the streamwise $\left(x_{1}\right)$, cross-stream $\left(x_{2}\right)$ and spanwise $\left(x_{3}\right)$ coordinates. The upper stream (subscript 1 for free-stream values) is nitrogen and the lower stream (subscript 2) is heptane. The layer is not symmetric in the $x_{2}$-direction, as we found in our simulations that the layer growth is considerably larger on the nitrogen side. The specified value of the initial flow Reynolds number,

$$
R e_{0}=\frac{0.5\left(\rho_{1}+\rho_{2}\right) \Delta U_{0} \delta_{\omega, 0}}{\mu_{R}}
$$

is chosen low enough to permit fully resolved simulations and is used to calculate the reference viscosity (in (2.24)). The free-stream density $\left(\rho_{1}\right.$ or $\left.\rho_{2}\right)$ is calculated for each pure species at its free-stream temperature $\left(T_{1}\right.$ or $\left.T_{2}\right)$ and at the initial uniform pressure $\left(p_{0}\right)$. The vorticity thickness is defined as $\delta_{\omega}(t)=\Delta U_{0} /\left(\partial U / \partial x_{2}\right)_{\max }$, where $U$ is the mean (or $\left(x_{1}, x_{3}\right)$-planar average) velocity in the streamwise direction, and $\Delta U_{0}=U_{1}-U_{2}$ is the velocity difference across the layer. Miller et al. (2001) explain the choice of the velocities of the two streams, the intent being to keep the vortices 
stationary in the computational domain. While the success in this endeavour was only partial, it proved that the choice of

$$
U_{1}=2 M_{c, 0} a_{s_{1}}\left[1+\left(\frac{a_{s_{1}}}{a_{s_{2}}}\right) \sqrt{\frac{\rho_{1} Z_{1}}{\rho_{2} Z_{2}}}\right]^{-1}, \quad U_{2}=-\sqrt{\frac{\rho_{1} Z_{1}}{\rho_{2} Z_{2}}} U_{1},
$$

for a real fluid was reasonable, where $M_{c, 0}$ is the initial Mach number and $Z=$ $p /\left(\rho T R_{u} / m\right)$ is the compression factor. The specification of $M_{c, 0}$ therefore determines $\Delta U_{0}$, and thus ultimately $\delta_{\omega, 0} \equiv \delta_{\omega}(0)$. The mean streamwise velocity is smoothed near the centreline using an error function profile, as are the mean temperature and mass fraction.

The simulations are started with streamwise and spanwise vorticity perturbations superimposed on the mean initial velocity profile:

$$
\begin{aligned}
& \omega_{1}\left(x_{2}, x_{3}\right)=F_{3 D} \frac{\lambda_{1} \Delta U_{0}}{\Gamma_{1}} f_{2}\left(x_{2}\right) f_{3}\left(x_{3}\right), \\
& \omega_{3}\left(x_{1}, x_{2}\right)=F_{2 D} \frac{\lambda_{3} \Delta U_{0}}{\Gamma_{3}} f_{1}\left(x_{1}\right) f_{2}\left(x_{2}\right),
\end{aligned}
$$

where $\Gamma_{1}$ and $\Gamma_{3}$ are the circulations,

$$
\begin{gathered}
f_{1}\left(x_{1}\right)=A_{0}\left|\sin \left(\frac{\pi x_{1}}{\lambda_{1}}\right)\right|+A_{1}\left|\sin \left(\frac{\pi x_{1}}{2 \lambda_{1}}\right)\right|+A_{2}\left|\sin \left(\frac{\pi x_{1}}{4 \lambda_{1}}\right)\right|+A_{3}\left|\sin \left(\frac{\pi x_{1}}{8 \lambda_{1}}-\frac{\pi}{2}\right)\right|, \\
f_{2}\left(x_{2}\right)=\exp \left[-\pi\left(\frac{x_{2}}{\delta_{\omega, 0}}\right)^{2}\right] \\
f_{3}\left(x_{3}\right)=B_{0} \sin \left(\frac{2 \pi x_{3}}{\lambda_{3}}\right)+B_{1} \sin \left(\frac{\pi x_{3}}{L_{3}}\right) .
\end{gathered}
$$

We use $F_{2 D}=0.1, A_{0}=1, A_{1}=0.5, A_{2}=A_{3}=0.35$ for the streamwise perturbations, and $F_{3 D}=0.05, B_{0}=1$ and $B_{1}=0.025$ for the spanwise perturbations. The wavelengths of the perturbations are $\lambda_{1}=7.29 \delta_{\omega, 0}$ (the most unstable wavelength for incompressible flow) and $\lambda_{3}=0.6 \lambda_{1}$, following Moser \& Rogers (1991). The initial vorticity thickness $\delta_{\omega, 0}$ is $6.859 \times 10^{-3} \mathrm{~m}$. The grid measures $0.2 \mathrm{~m} \times 0.232 \mathrm{~m} \times 0.12 \mathrm{~m}$ for all simulations, to accommodate four wavelengths in the streamwise and spanwise directions, and the evolution of the layer encompasses roll-up and two pairings of the initial spanwise vortices. The larger than typical streamwise vorticity forcing amplitude (5\%, instead of $2.25 \%$ as in Miller \& Bellan 2000) was necessary to entrain the heavy heptane $\left(\left(\rho_{2} / \rho_{1}\right)_{0}=12.88\right.$ for the conditions of the simulations $)$ and achieve transition.

Periodic boundary conditions are used for the streamwise and spanwise directions, and non-reflecting outflow conditions are used in the cross-stream direction. The real gas outflow conditions, derived by Okong'o \& Bellan (2002) and akin to the perfect gas conditions of Poinsot \& Lele (1992) and Baum, Poinsot \& Thévenin (1994), are essential since the initial perturbation causes large pressure waves (about $10 \%$ above the mean pressure of $60 \mathrm{~atm}$ ) which must be allowed out of the domain with minimal reflection. 


\begin{tabular}{rcccc}
\hline Run & $R e_{0}$ & $N_{1} \times N_{2} \times N_{3}$ & $t_{\text {trans }}^{*}$ & $R e_{m, \text { trans }}$ \\
R400 & 400 & $192 \times 224 \times 112$ & 150 & 972 \\
R500 & 500 & $240 \times 288 \times 144$ & 155 & 1250 \\
R600 & 600 & $288 \times 336 \times 176$ & 135 & 1452 \\
TABLE 1. Simulation parameters. All runs have four initial vortices and two pairings, $\alpha_{I K}=0.1$, \\
$M_{c, 0}=0.4, T_{2}=600 \mathrm{~K}, T_{1}=1000 \mathrm{~K}$, and $p_{0}=60 \mathrm{~atm}\left(\left(\rho_{2} / \rho_{1}\right)_{0}=12.88\right)$. \\
\hline
\end{tabular}

\section{Numerics}

The conservation equations were solved numerically using a fourth-order explicit Runge-Kutta time integration and a sixth-order compact scheme for spatial derivatives (Kennedy \& Carpenter 1994). Time stability was achieved by following the advice of M. Carpenter (2000, personal communication) and filtering the conservative variables every ten time steps in the interior, in each spatial direction alternately, using an eighth-order filter (Kennedy \& Carpenter 1994). Since high-order boundary filters were unstable, no filtering was applied at the non-periodic $\left(x_{2}\right)$ boundaries. The computations were parallelized using three-dimensional domain decomposition and message passing. The tridiagonal solver for the compact derivative scheme was efficiently parallelized using the method of Muller \& Scheerer (1991).

In our solution protocol, once the temperature, density and mass fractions are computed, the pressure is calculated from the EOS. However, the numerical solution is obtained for the energy rather than the temperature. To efficiently calculate the temperature from the known energy, density and mass fraction, we use a highly accurate fit (details in the Appendix).

\section{Results}

Our simulations pursue several goals. First, we want to achieve a mixing transition state for each simulation. By our stringent criterion for mixing transition, not only must the momentum-thickness-based Reynolds number, $R e_{m}=R e_{0} \delta_{m} / \delta_{\omega, 0}$ be 'large enough' (see below for the definition of $\delta_{m}$ ), but we must also see other manifestations of transition such as dramatically increased product thickness, $\delta_{p}$, (see definition below), continuous increase in enstrophy, development and abrupt increase in the volume-averaged positive spanwise vorticity (due to the form of the initial mean velocity profile, all initial spanwise vorticity is negative), and the somewhat heuristic test of Lin \& Corcos (1984) who suggest that a 'collapse parameter' exceeding a threshold is indicative of transition. The discussion on the mixing layer evolution presented below shows that these criteria have been met in all simulations. Table 1 lists the initial conditions of the simulations performed herein.

Second, we want to analyse and characterize the detailed features of the supercritical transitional state, particularly aspects that are different from those of the much studied temporal gaseous layers. Although lacking an experimental database for direct comparison with our simulations (current experimental studies of supercritical temporal mixing layers do not exist, and we aware of only the density-stratified mixing layer experiments of Atsavapranee \& Gharib 1997), we are interested in identifying distinctive optical features that might have also been observed in spatial supercritical mixing layers or jets. As mentioned in the Introduction, Metcalfe et al. (1987) point out that there are optical features common to spatial and temporal turbulent shear flows. 
The third goal of our work is the identification of the physical origin of dissipation at the transitional state. Whereas in gaseous flows it is recognized that the dissipation is due to viscous stress effects (e.g. Liu, Katz \& Meneveau 1999), this may not necessarily be the case under supercritical conditions, owing to the additional terms and associated transport coefficients in the transport matrix. In fact, Okong'o \& Bellan (2000) have derived the entropy equation and the form of the dissipation for a supercritical fluid and have shown that, in addition to the viscous dissipation, there are contributions due to mass and temperature transport.

Finally, as assumed PDF modelling is a well-accepted method for describing turbulent flows (e.g. O'Brien 1980; Pope 1985; Frankel et al. 1993; Dopazo 1994), we are also interested in the PDF form of the dynamic and thermodynamic variables at the transitional state. Therefore, our fourth goal is the identification of the PDFs at the DNS scale, as well as at a larger scale that might be envisaged for modelling domains at the spatial scales of practical applications. We wish to explore whether these PDFs are close to known forms used in gaseous flow PDF modelling such as Gaussian or $\beta$ densities. If they emulate the shape of known PDFs, modelling of supercritical turbulent flows could be developed following existing methods and benefit from available codes. The last issue of interest is the evaluation of the current typical PDF approach to reactive flow modelling whereby the reaction rate, a joint temperature-species (partial densities) PDF, is usually represented through the product of the marginal PDFs. Because our DNS does not include reactions, it represents a more favourable case for such an approach than in equivalent reacting flow DNS since the addition of reaction will necessarily correlate the temperature and the partial densities.

\subsection{Mixing layer evolution}

One of the fundamental quantities characterizing a mixing layer is its growth. Although the definition of layer growth is not unique, the quantity that is most often used to measure it is the vorticity thickness, $\delta_{\omega}$. However, the fluctuations exhibited by $\delta_{\omega}$ make it sometimes an unreliable measurement of growth. It is thus preferable to measure the layer growth by the momentum thickness

$$
\delta_{m}=\frac{1}{\left(\theta_{1}-\theta_{2}\right)^{2}} \int_{-L_{2, \text { min }}}^{L_{2, \max }}-\left(\theta_{2}+\left\langle\rho u_{1}\right\rangle\right)\left(\theta_{1}+\left\langle\rho u_{1}\right\rangle\right) \mathrm{d} x_{2},
$$

with $\theta_{1}=\left\langle\rho u_{1}\right\rangle_{x_{2}=L_{2, \max }}$ and $\theta_{2}=\left\langle\rho u_{1}\right\rangle_{x_{2}=-L_{2, \min }}$, where \langle\rangle denotes $\left(x_{1}, x_{3}\right)$ plane averaging, $L_{2, \max }=-L_{2} / 3$ and $L_{2, \min }=2 L_{2} / 3$. Because $\delta_{m}$ is an integral quantity, it does not exhibit the fluctuations inherent in $\delta_{\omega}$. While the growth is primarily a consequence of entrainment, the product thickness defined as

$$
\delta_{p}=\iiint_{V} \rho Y_{p} \mathrm{~d} V
$$

in mass units, where $Y_{p}=2 \min \left(Y_{h}, Y_{n}\right)$, is a direct consequence of molecular mixing as explained by Cortesi et al. (1999). Both $\delta_{m} / \delta_{\omega, 0}$ and $\delta_{p} / \delta_{p, 0}$ are illustrated in figures $2(a)$ and $2(b)$, respectively, for R400, R500 and R600 (see table 1) as a function of the non-dimensional time $t^{*}=t \Delta U_{0} / \delta_{\omega, 0}$; the only difference between the three simulations is the value of the reference viscosity. For all runs, $\delta_{m} / \delta_{\omega, 0}$ and $\delta_{p} / \delta_{p, 0}$ display a gradual increase up to the first pairing, with indistinguishable momentum thickness growth among runs. This latter aspect is consistent with the fact that, at this early stage of layer evolution, the entrainment is unaffected by the weak mixing; it is only in the further stage of layer development that the mixing 

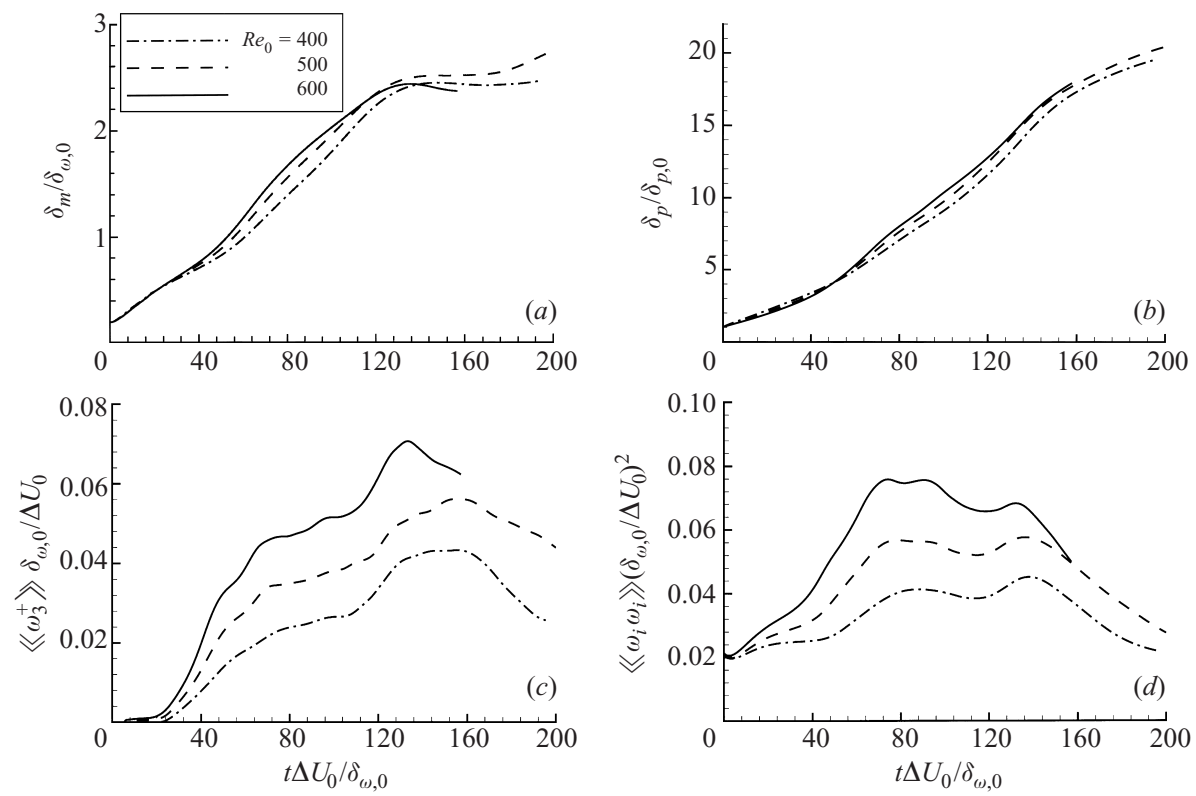

FIGURE 2. Time evolution of global quantities for $R e_{0}=400, R e_{0}=500$ and $R e_{0}=600$ :

$(a)$ momentum thickness, $(b)$ product thickness, $(c)$ positive spanwise vorticity, $(d)$ enstrophy.

becomes $R e_{0}$ specific. From the first pairing, the growth rate increases to the time of transition, $t^{*}=150$ for R400, $t^{*}=155$ for R500 and $t^{*}=135$ for R600. At transition, $R e_{m}$ is 972 for $\mathrm{R} 400,1250$ for R500, and 1452 for R600, clearly similar in magnitude to the transition $R e_{m}$ obtained by Miller \& Bellan (2000) for a drop-laden shear layer and exceeding that of Moser \& Rogers (1993) for gaseous layers. The slow decline of $\delta_{m} / \delta_{\omega, 0}$ past transition is due to the forcing, and is in fact only a temporary plateau as the layer continues to grow, although at a reduced rate. This oscillatory growth rate is in agreement with previous findings from gaseous flow DNS showing 'truly' self-similar flow profiles only for unforced, turbulent initial conditions, leading to a linear layer growth rate (see Moser \& Rogers 1991; Slessor, Bond \& Dimotakis 1998). The product thickness is a monotonically increasing function of $t^{*}$, indicating that molecular mixing proceeds unabated.

Since vorticity, $\omega \equiv \nabla \times \boldsymbol{u}$, plays a major role in turbulent flows (see Chorin 1994), we also examined global features of the flow intimately associated with $\omega$. In figures $2(c)$ and $2(d)$ the volume-averaged non-dimensional positive spanwise vorticity, $\left\langle\left\langle\omega_{3}^{+}\right\rangle\right\rangle \delta_{\omega, 0} / \Delta U_{0}$, and the non-dimensional enstrophy, $\left\langle\left\langle\omega_{i} \omega_{i}\right\rangle\right\rangle\left(\delta_{\omega, 0} / \Delta U_{0}\right)^{2}$, evolutions are depicted as functions of $t^{*}$; here $\langle\langle\rangle\rangle$ denotes volume averaging. The positive spanwise vorticity (figure $2 c$ ), initially null, increases immediately after the layer roll-up $\left(t^{*}=25\right)$, and grows at an accelerated rate during the entrainment period leading to the first pairing. Further augmentation of $\left\langle\left\langle\omega_{3}^{+}\right\rangle\right\rangle \delta_{\omega, 0} / \Delta U_{0}$ is the competing result of entrainment and pairing which destabilizes the layer, and the formation of large- $|\nabla \rho|$ regions (see below) which stabilizes the layer. For all runs, $\left\langle\left\langle\omega_{3}^{+}\right\rangle\right\rangle \delta_{\omega, 0} / \Delta U_{0}$ reaches maximum at transition, although as expected its value is largest for R600. Chorin (1994) states that the most important consequence of vortex motion in three dimensions is vortex stretching and the resulting lack of conservation of vorticity and enstrophy. Since it is generally believed that stretching increases the enstrophy, the variation of the enstrophy is a direct measure of this essential mechanism of 

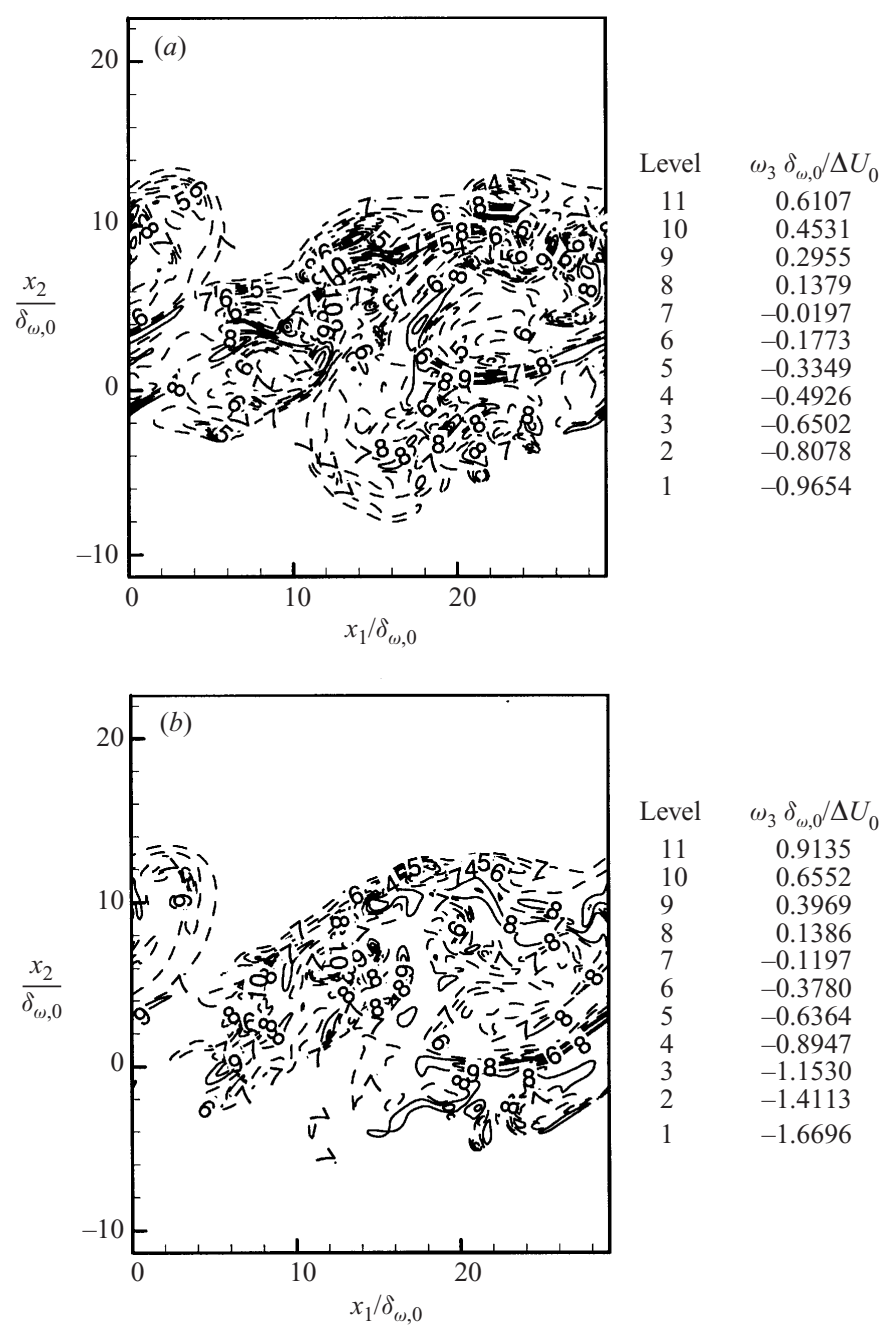

FIGURE 3. Spanwise vorticity for $R e_{0}=600$ at $t^{*}=135$ in $(a)$ braid plane, $(b)$ between-the-braid plane. Dashed lines are negative values, solid lines are positive values.

turbulence production. Illustrated in figure $2(d)$, the enstrophy displays an increase, presenting evidence of vortex stretching and turbulence production.

Supporting the global information on the production of positive spanwise vorticity, figures $3(a)$ and $3(b)$ portray the spanwise vorticity for R600 at $t^{*}=135$ in the braid plane $\left(x_{3} / L_{3}=1 / 16\right)$ and the between-the-braid plane $\left(x_{3} / L_{3}=1 / 2\right)$. The creation of positive spanwise vorticity, plotted in solid lines, implies small-scale production.

Streamwise and spanwise energy spectra for all velocity components are displayed in figure 4 for R600 at the transitional state, demonstrating the full resolution of the simulation for the highest $R e_{m}$ achieved. Clearly, most of the energy is in the low-wavenumber regime, with the amount of energy in the high wavenumbers being at least eight, and as much as ten, orders of magnitude smaller, and there is no accumulation of energy at the high wavenumbers, all of which indicates that the computation is well-resolved (see Kim, Moin \& Moser 1987). The peak in the spanwise energy spectra at a wavenumber of 4 can be attributed to the spanwise forcing. 

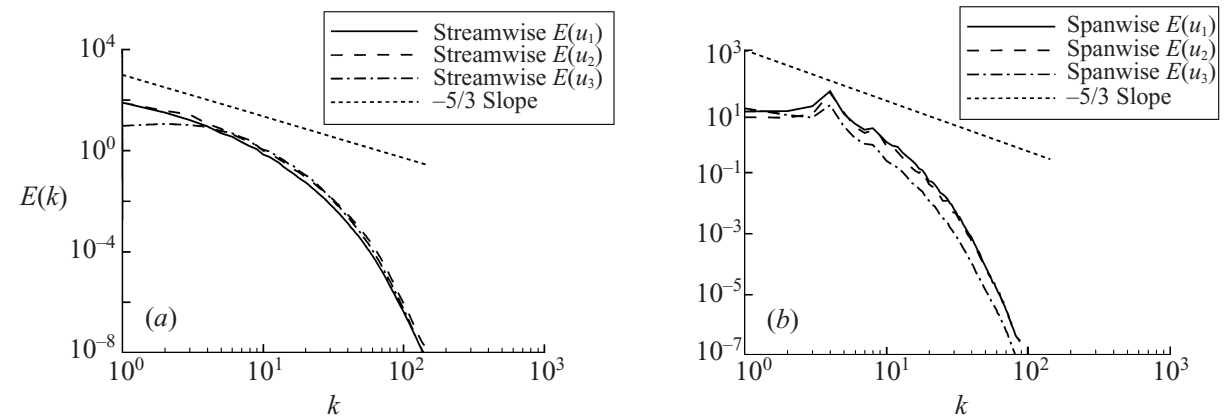

FIGURE 4. One-dimensional energy spectra for $R e_{0}=600$ at $t^{*}=135$ : $(a)$ streamwise spectra, (b) spanwise spectra.
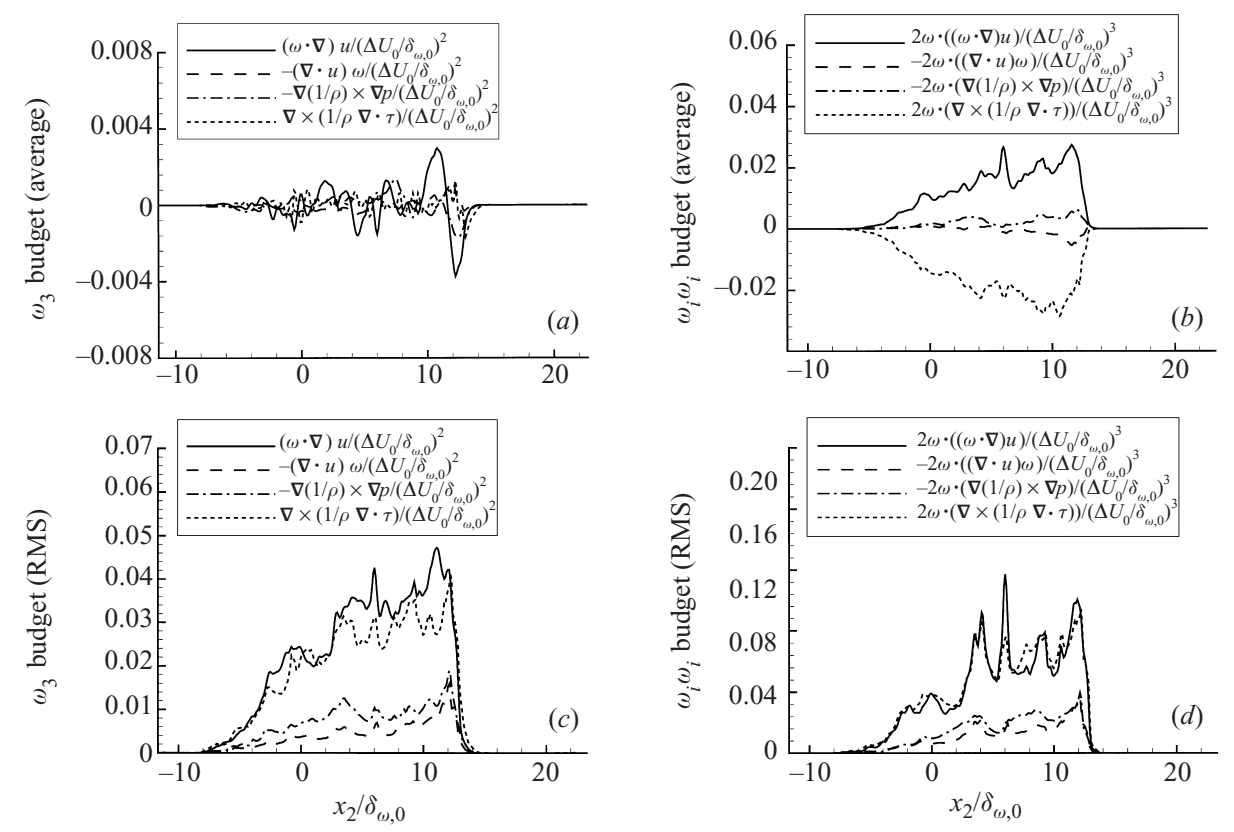

FIGURE 5. Vorticity budgets for $R e_{0}=600$ at $t^{*}=135:(a)$ average $\omega_{3},(b)$ average $\omega_{i} \omega_{i}$, (c) RMS $\omega_{3},(d)$ RMS $\omega_{i} \omega_{i}$.

To further identify the phenomena predominantly responsible for vorticity production, we considered the budget of the vorticity equation

$$
\frac{\mathrm{D} \boldsymbol{\omega}}{\mathrm{D} t}=(\omega \cdot \nabla) \boldsymbol{u}-(\nabla \cdot \boldsymbol{u}) \boldsymbol{\omega}-\nabla\left(\frac{1}{\rho}\right) \times \nabla p+\nabla \times\left(\frac{1}{\rho} \nabla \cdot \tau\right)
$$

where $\mathrm{D} / \mathrm{D} t$ is the substantial derivative, and of the vorticity-magnitude-squared equation

$$
\frac{\mathrm{D}\left(\omega^{2}\right)}{\mathrm{D} t}=2 \omega \cdot(\omega \cdot \nabla) \boldsymbol{u}-2(\nabla \cdot \boldsymbol{u}) \omega^{2}-2 \omega \cdot \nabla\left(\frac{1}{\rho}\right) \times \nabla p+2 \omega \cdot \nabla \times\left(\frac{1}{\rho} \nabla \cdot \tau\right) .
$$

Homogeneous $\left(x_{1}, x_{3}\right)$-plane averages of the spanwise vorticity and the vorticity magnitude squared for R600, illustrated in figures $5(a)$ and $5(b)$, respectively, show that on average stretching/tilting dominates all other effects in the production of $\omega_{3}$, 
and that the viscous term generally makes the major contribution to the vorticity magnitude, although at some cross-stream locations the stretching and tilting term may exceed the viscous contribution. These results resemble those of the layer without transition (see Miller et al. 2001), with the exception that the stretching and tilting contribution is entirely positive in the latter. For the mixing layer of Miller et al. (2001) that did not transition, the primary phenomenon is the emergence of turbulent scales being produced through stretching and tilting, whereas for the present layers, which did reach transition, the primary phenomenon is turbulence destruction through the action of viscosity. This conceptual picture is supported by the dominance of the viscous terms in the production of average vorticity magnitude. The spiky aspect of the stretching and tilting average is evidence of the concentrated action of this term at the boundary of the coherent vortices which it mostly maps (not shown). In contrast, most of the action of the viscous terms is inside the coherent vortices where the small-scale formation occurs. Equivalent root-mean-square (RMS) plots, figures 5(c) and $5(d)$, exhibit the dominance of the stretching/tilting, followed by the viscous, baroclinic and dilatation effects for both $\omega_{3}$ and the vorticity magnitude squared. The predominance of stretching/tilting in the RMS, even for the vorticity magnitude, is due to the continuing production of turbulence by the action of $3 \mathrm{D}$ effects. It is clear that most of the vorticity is produced on the nitrogen side of the layer, it being the lighter fluid.

Lin \& Corcos (1984) derived a parameter measuring the vorticity focusing of streamwise vortex sheets into concentrated round vortices, which they called the 'collapse' parameter. Based on their incompressible analysis, this collapse parameter must surpass the critical value of 13 in order for a layer to achieve mixing transition. An examination of this collapse parameter value reveals that it is 31 at $t^{*}=150$ for R400, 43 at $t^{*}=155$ for R500, and is 55 at $t^{*}=135$ for R600, adding a more heuristic confirmation of transition attainment to the quantitative analysis presented above.

\subsection{Visualizations of the thermodynamic variables at the transitional state}

Flow visualizations usually reveal aspects that may be spatially evident, but may disappear upon averaging. Moreover, these visualizations might be essential in identifying the distinct optical features common to temporal and spatial mixing layers.

In a previous study, Miller et al. (2001) have illustrated $|\nabla \rho|$ for a layer that did not reach transition and identified concentrated regions where $|\nabla \rho|$ reached large values. The qualitative comparison between these regions and the optical observations of Chehroudi et al. (1999) who experimentally identified 'wisps' of fluid emerging from a supercritical jet injected into a supercritical medium were encouraging. The question is whether the same qualitative features may be identified at transition, and if so what is the large- $|\nabla \rho|$ region composition and thermodynamic state. To explore these aspects, $|\nabla \rho|, Y_{h}$ and $\alpha_{D}$ are displayed in figures $6(a, b), 6(c, d)$, and $6(e, f)$, respectively, in the braid and the between-the-braid planes. Not only are the regions of concentrated large $|\nabla \rho|$ values again evident, but they also appear considerably more convoluted than in the study of Miller et al. (2001), owing to the turbulent scales distorting the large- $|\nabla \rho|$ regions formed as a result of entrainment and mixing. The heptane mass fraction contours show parcels of heptane fluid that have "broken off' from the original stream and have been transported towards the upper nitrogen stream. The mixing region thus formed contains non-ideal fluid $\left(\alpha_{D}<1\right)$ as shown in 

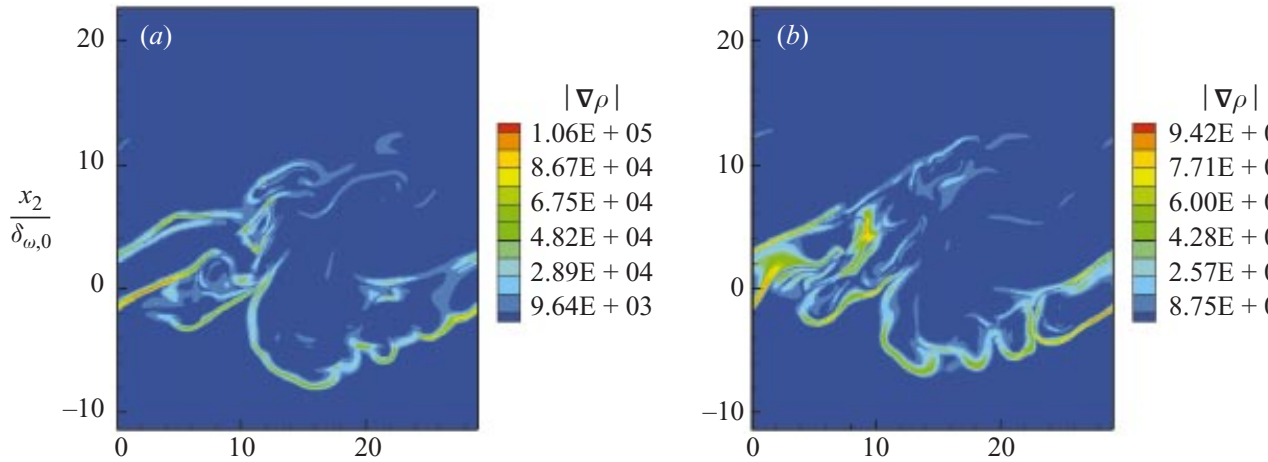

$9.42 \mathrm{E}+04$

$7.71 \mathrm{E}+04$

$6.00 \mathrm{E}+04$

$4.28 \mathrm{E}+04$

$2.57 \mathrm{E}+04$

$8.75 \mathrm{E}+03$
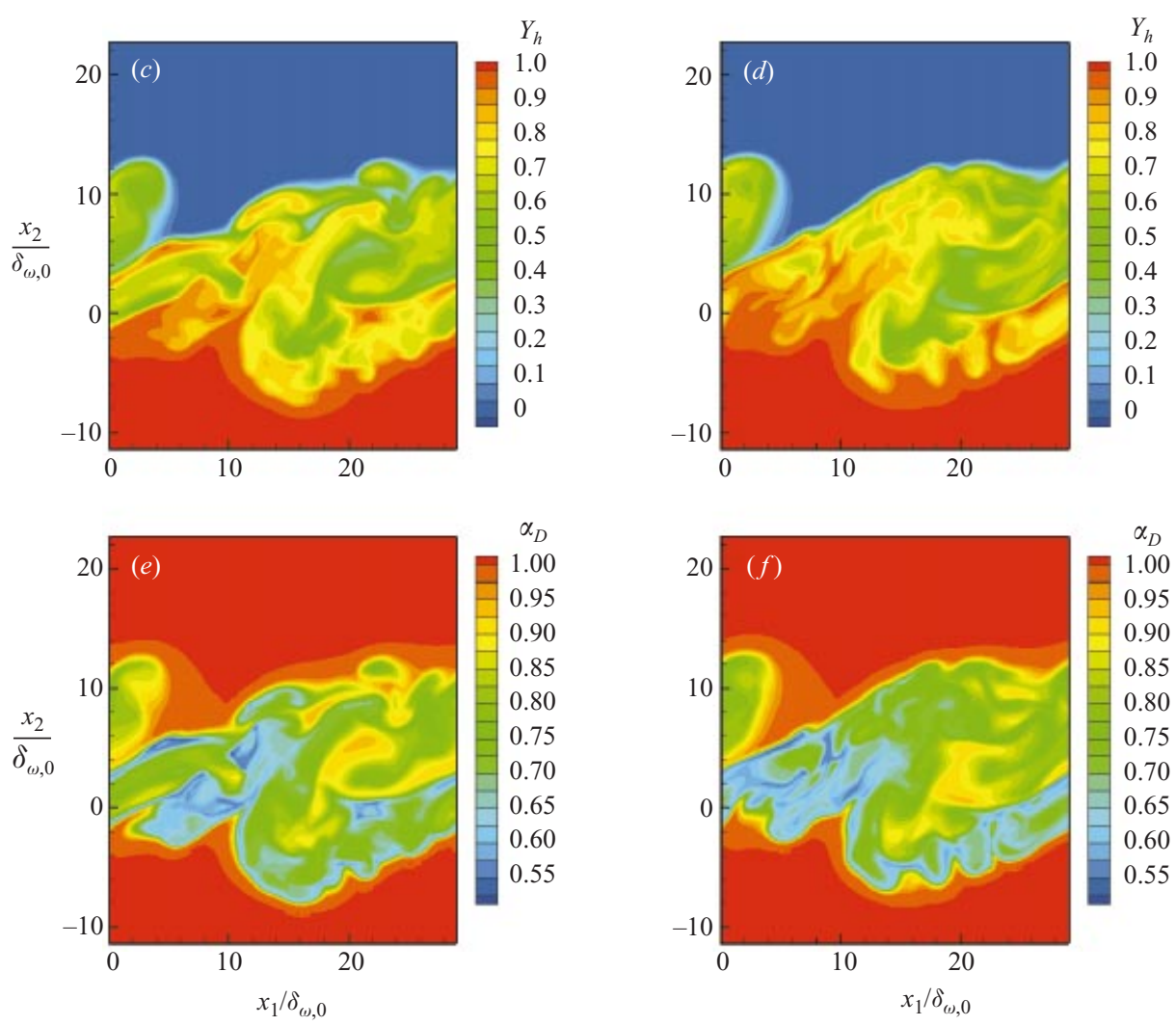

FIGURE 6. Contours of $(a, b)$ density gradient magnitude, $(c, d)$ heptane mass fraction and $(e, f)$ mass diffusion factor in braid plane $(a, c, e)$ and between-the-braid plane $(b, d, f)$ for $R e_{0}=600$ at $t^{*}=135$.

figures $6(e)$ and $6(f)$, whereas the two regions of unvitiated species behave as ideal mixtures.

The striking visual correlation between the regions of large $|\nabla \rho|$, disintegrated $Y_{h}$ and minimal $\alpha_{D}$ provided the motivation to further investigate the composition of the fluid in these regions and its degree of mixing. Conditional averages for both braid and between-the-braid planes are listed in tables 2 and 3, and show that the fluid in the large- $|\nabla \rho|$ regions is mostly heptane, but that nitrogen has dissolved into it. This fluid has difficulty in mixing at the molecular level, evidenced by the lower 


$\begin{array}{ccc}\text { Cutoff } & \text { Average } Y_{h} & \text { Average } \alpha_{D} \\ 0.3|\nabla \rho|_{\max }=3.47 \times 10^{4} & 0.904 & 0.645 \\ 0.2|\nabla \rho|_{\max }=2.31 \times 10^{4} & 0.887 & 0.658 \\ 0.1|\nabla \rho|_{\max }=1.16 \times 10^{4} & 0.840 & 0.688\end{array}$

TABLE 2. Conditional averages of heptane mass fraction and of mass fraction diffusion factor over regions where $|\nabla \rho|>$ cutoff. The calculations are made in the braid plane at $t^{*}=135$ for R600.

$\begin{array}{ccc}\text { Cutoff } & \text { Average } Y_{h} & \text { Average } \alpha_{D} \\ 0.3|\nabla \rho|_{\text {max }}=3.08 \times 10^{4} & 0.921 & 0.638 \\ 0.2|\nabla \rho|_{\text {max }}=2.06 \times 10^{4} & 0.910 & 0.649 \\ 0.1|\nabla \rho|_{\text {max }}=1.03 \times 10^{4} & 0.869 & 0.679\end{array}$

TABLE 3. As table 2 but for calculations in the between-the-braid plane.

\begin{tabular}{lccc}
\hline & Braid plane & Between-the-braid plane & Global \\
$Y_{h},|\nabla \rho|$ Correlation & 0.30 & 0.32 & 0.31 \\
$\alpha_{D},|\nabla \rho|$ Correlation & -0.60 & -0.62 & -0.62 \\
$|\nabla \rho|_{\text {max }}$ & $1.16 \times 10^{5}$ & $1.03 \times 10^{5}$ & $1.35 \times 10^{5}$ \\
& TABLE 4. Correlations with $|\nabla \rho|$ at $t^{*}=135$ for R600. \\
\hline
\end{tabular}

than unity value of $\alpha_{D}$. With increased cutoff value for the proportion of $|\nabla \rho|_{\max }$ on which the $Y_{h}$ and $\alpha_{D}$ averages are conditioned, the fluid composition becomes closer to heptane, and the molecular mixing becomes increasingly hindered. Finally, a plane-based correlation

$$
\mathscr{R}(\mathscr{X}, \mathscr{Y})=\frac{\langle\mathscr{X} \mathscr{Y}\rangle-\langle\mathscr{X}\rangle\langle\mathscr{Y}\rangle}{\sqrt{\left(\left\langle\mathscr{X}^{2}\right\rangle-\langle\mathscr{X}\rangle^{2}\right)\left(\left\langle\mathscr{Y}^{2}\right\rangle-\langle\mathscr{Y}\rangle^{2}\right)}}
$$

listed in table 4 for R600, where $\mathscr{X}$ and $\mathscr{Y}$ are generic variables, confirms the conditional-average results. $|\nabla \rho|$ and $Y_{h}$ are only moderately correlated, whereas $|\nabla \rho|$ and $\alpha_{D}$ are more strongly correlated and while $|\nabla \rho|$ increases, $\alpha_{D}$ decreases. The information obtained so far indicates that the regions of large $|\nabla \rho|$ are formed as a result of two different processes. The first process is dynamic and results in the convolution of the original density boundary. The second process is a combination of thermodynamics and transport mechanisms, through solvation and mass diffusion, respectively. Both of these processes determine the structure of the large- $|\nabla \rho|$ regions at the transitional state.

Figure 7 depicts the compression factor and shows that the mixture exhibits larger departures from a perfect gas with increasing cross-stream penetration into the lower, heptane stream. In addition to the strong departures from mixture ideality, these considerable departures from perfect gas behaviour indicate that the real gas EOSs are essential in the mathematical description of the present situation. From the numerical method viewpoint, this highlights the importance of characteristic wave boundary conditions based upon real gas EOSs, particularly on the heptane side where $Z \simeq 0.5$ ( $Z=1$ for a perfect gas).

Since the layer region contains a mixture of fluids, and because the critical point of a mixture is a function of its composition, we investigated the thermodynamic 

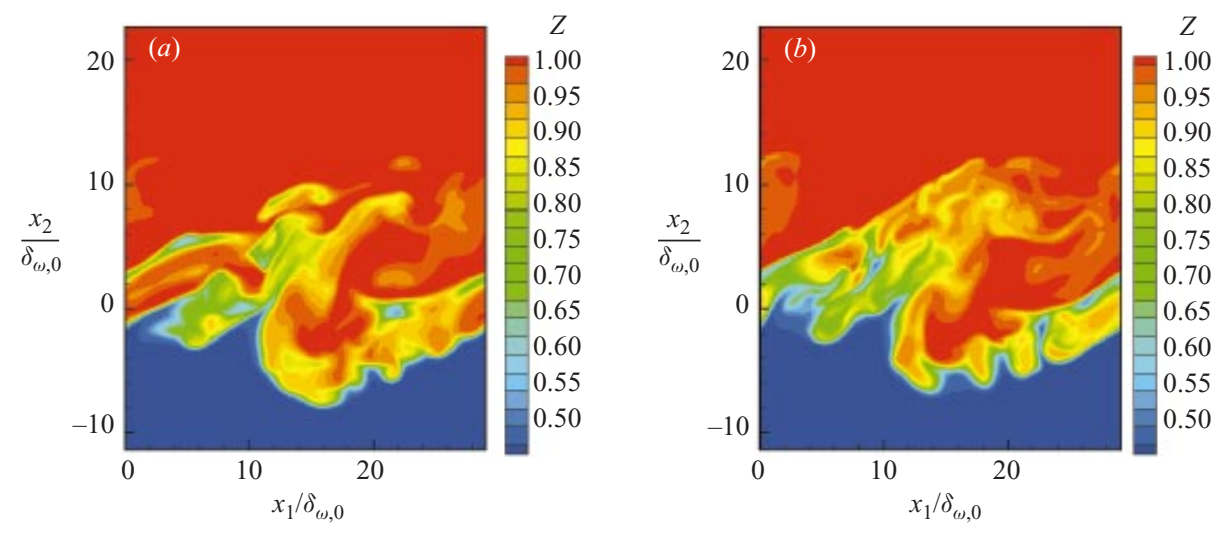

FIGURE 7. Contours of compression factor for $R e_{0}=600$ at $t^{*}=135$ in $(a)$ braid plane,

(b) between-the-braid plane.

state of the mixture in both braid and between-the-braid planes. Calculated here according to Peng \& Robinson (1977) and plotted in figure 8(a), the critical point occurs when both the determinant of the second derivatives of $G,|\boldsymbol{U}|$, and that of the second derivatives of $G$ combined with the first derivatives of $|\boldsymbol{U}|$ are null. Since

$$
\alpha_{D \alpha \beta}=\beta X_{\alpha}\left(\partial^{2} G / \partial X_{\alpha} \partial X_{\beta}\right)_{p, T, X_{\gamma(\gamma \neq \alpha, \beta)},},
$$

a necessary condition for attaining the critical point for a binary species system is that $\alpha_{D}=0$. Note that the critical temperature, $T_{c}$, has a maximum of $540 \mathrm{~K}$, whereas the critical pressure, $p_{c}$, has a maximum of $8735 \mathrm{~atm}$; therefore at our initial conditions of $T_{1}=1000 \mathrm{~K}, T_{2}=600 \mathrm{~K}$ and $p=60 \mathrm{~atm}$, the temperature is supercritical everywhere, and the pressure is subcritical for $0.01<Y_{h}<0.92$. Since $\alpha_{D}>0$ in figures 6(e) and $6(f)$, preliminary indications are that the critical point is not reached. These indications are confirmed by illustrations of $T-T_{c}$ and $p-p_{c}$ in figures $8(b)$ and $8(c)$ respectively; only the braid-plane contours are shown, the between-the-braid-plane contours being similar. Indeed, when $p>p_{c}$ or $T>T_{c}$, in the $(p, T)$-plane there is no longer the possibility of a two-phase (e.g. gas/liquid) region and instead there is only a single-phase region (see discussion in Harstad \& Bellan 2001). Clearly, since $\left(T-T_{c}\right)>0$ everywhere, the fluid is supercritical in the entire plane. It is noteworthy that $p-p_{c}$ displays both positive and negative regions with the null contour mapping the coherent vortices, which contain the mixed fluid. Outside these vortices, $\left(p-p_{c}\right)>0$, conforming to the free-stream conditions. On the other hand, inside the vortices there is a region of high vorticity, low pressure. The fact that the pressure level fell from supercritical in the free stream to below the critical pressure inside the vortices is due to the change in composition which led to a change in the local critical pressure.

\subsection{Irreversible entropy production (dissipation)}

The entropy production is a quantity of great interest because it contains a measure of the important phenomena determining, in particular, the fate of a turbulent flow. Entropy production may be of the reversible type, coming from fluxes at the boundary of the domain under consideration, or of the irreversible type; the irreversible entropy production is in fact the dissipation. Information about the dissipation can be useful in many ways, one of which is the modelling of the small-scale behaviour; for example, because it is precisely the small-scale dissipation which must be duplicated by subgrid 

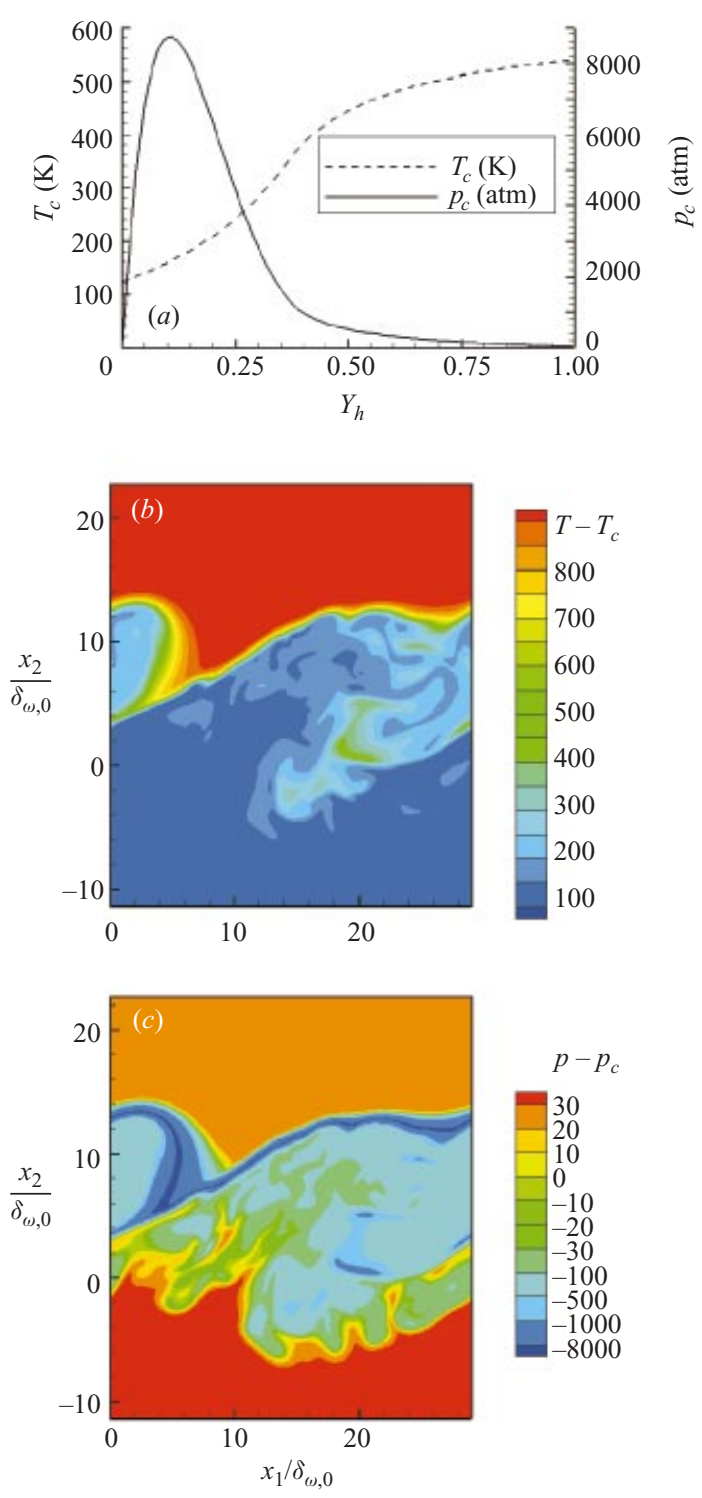

FiguRE 8. (a) Critical temperature and critical pressure vs. heptane mass fraction for heptane-nitrogen mixtures; $(b)$ temperature - critical temperature and $(c)$ pressure - critical pressure in braid plane for $R e_{0}=600$ at $t^{*}=135$.

scale (SGS) models in large-eddy simulations, Liu et al. (1999) used this information to model the SGS viscous stresses.

Okong'o \& Bellan (2000) derived the entropy equation for a supercritical flow and showed that if $g$ denotes the rate of irreversible entropy production, then

$$
\begin{gathered}
g=g_{v i s c}+g_{\text {temp }}+g_{\text {mass }}, \\
g_{v i s c}=\frac{\mu}{T}\left(2 S_{i j} S_{i j}-\frac{2}{3} S_{k k} S_{l l}\right), \quad g_{\text {temp }}=\frac{\lambda}{T^{2}} \frac{\partial T}{\partial x_{j}} \frac{\partial T}{\partial x_{j}}, \\
g_{\text {mass }}=\frac{1}{Y_{n} Y_{h} \rho D} \frac{R_{u} m}{m_{n} m_{h}} j_{h j} j_{h j},
\end{gathered}
$$




\begin{tabular}{cccc}
\hline$t^{*}$ & Braid plane & Between-the-braid plane & Global \\
105 & 0.526 & 0.170 & 0.335 \\
120 & 0.533 & 0.524 & 0.394 \\
135 & 0.506 & 0.453 & 0.416 \\
& \multicolumn{4}{c}{ TABLE 5. Correlations between $|\nabla \rho|$ and $g$ for R600. } \\
\hline
\end{tabular}

where according to (2.17) and (2.18), $g_{\text {mass }}$ contains the departure from mixture nonideality, $\propto \alpha_{D}$, and the Soret term, $\propto \alpha_{B K}$, (through $j_{h j}$ ). The question arises of the importance of each of $g_{v i s c}, g_{\text {temp }}$ and $g_{\text {mass }}$ at various stages of the transitional supercritical mixing layer evolution and of possible correlations of high g-activity regions with the regions of large $|\nabla \rho|$ which seem to play a pivotal role in determining the characteristics of the layer transitional state. To explore these topics, we calculate the $g_{\text {visc }}, g_{\text {temp }}, g_{\text {mass }}$ and $g$ volume averages, RMS, and their small-scale contribution. The small-scale contribution is calculated by filtering the DNS solution with a cubic top-hat filter having a filter width $\Delta=4 \max \left(\Delta x_{1}, \Delta x_{2}, \Delta x_{3}\right)$, where $\Delta x_{i}$ is the computational grid spacing, and calculating the difference between the unfiltered and filtered solution. These calculations were performed at two times past the second pairing but prior to transition, and at transition; e.g. for R600 the selected times were 105,120 and 135. For each of the $g$ terms, $\left(x_{1}, x_{3}\right)$-plane averages were plotted (not shown) both at the DNS and filtered scales. Also, visualizations of $g$ and $|\nabla \rho|$ both in the braid and between-the-braid planes (not shown) were scrutinized for possible visual correlations. Contour plots of the dissipation illustrated the occurrence of strong dissipation almost exclusively within the regions of large $|\nabla \rho|$. This finding is in agreement with the experimental results of Hannoun, Fernando \& List (1988) who observed that a density interface is very effective at damping turbulent eddies, which upon impingement 'bounce' back without significant entrainment of unstirred fluid, instead of overturning. The present dissipation plots displayed a topologically continuous aspect on the nitrogen side, whereas on the heptane side, pockets of high dissipation were exhibited. To quantify these visualizations, the plane correlation between $g$ and $|\nabla \rho|$ was calculated and is listed in table 5. The results show that the variables are moderately correlated in both the braid and between-the-braid planes, and that the global correlation coefficient increases as the layer evolves towards transition.

Tables 6 and 7 contain the values of the unfiltered and SGS domain-averaged irreversible entropy production for R600; the results are typical of all simulations. The species mass flux contribution dominates both the unfiltered and the SGS by a factor of 5 over the next larger $g_{\text {temp }}$ contribution, consistent with the large effective Lewis number under supercritical conditions (see Harstad \& Bellan 1999). The ratios $g_{\text {mass }} / g$ and $g_{\text {mass }}^{S G S} / g^{S G S}$ displayed in tables 6 and 7, respectively, show that approximately $77 \%$ of the average and small-scale dissipation at $t^{*}=135$ is due to species mass fluxes, with a percentage only marginally larger at the previous two times. $g_{\text {temp }}$ represents at most $17 \%$ of $g$, and $g_{\text {temp }}^{S G S} / g^{S G S}$ is at most $16.4 \%$. The smallest contribution is that of $g_{v i s c}$ (the maximum being just in excess of $6 \%$ ), in sharp departure from atmospheric-pressure mixing layers that are dominated by viscous effects (e.g. Moser \& Rogers 1993). Table 8 documents the domain average ratio of the small scales of each of $g_{\text {visc }}, g_{\text {temp }}, g_{\text {mass }}$ to the average $g$ and to their average respective contribution (i.e. $g_{v i s c}, g_{\text {temp }}, g_{\text {mass }}$ ). Clearly, a substantial amount of the total 


\begin{tabular}{|c|c|c|c|c|c|c|}
\hline & \multicolumn{2}{|c|}{$t^{*}=105$} & \multicolumn{2}{|c|}{$t^{*}=120$} & \multicolumn{2}{|c|}{$t^{*}=135$} \\
\hline & $\left(\mathrm{W} \mathrm{m} \mathrm{m}^{-3} \mathrm{~K}^{-1}\right)$ & $g_{k} / g$ & $\left(\mathrm{~W} \mathrm{~m} \mathrm{~m}^{-3} \mathrm{~K}^{-1}\right)$ & $g_{k} / g$ & $\left(\mathrm{~W} \mathrm{~m}^{-3} \mathrm{~K}^{-1}\right)$ & $g_{k} / g$ \\
\hline$g_{v i s c}$ & $8.20 \times 10^{4}$ & 0.065 & $7.73 \times 10^{4}$ & 0.058 & $7.91 \times 10^{4}$ & 0.062 \\
\hline$g_{\text {temp }}$ & $1.97 \times 10^{5}$ & 0.157 & $2.22 \times 10^{5}$ & 0.166 & $2.18 \times 10^{5}$ & 0.170 \\
\hline$g_{\text {mass }}$ & $9.74 \times 10^{5}$ & 0.778 & $1.04 \times 10^{6}$ & 0.777 & $9.83 \times 10^{5}$ & 0.768 \\
\hline$g$ & $1.25 \times 10^{6}$ & 1.000 & $1.34 \times 10^{6}$ & 1.000 & $1.28 \times 10^{6}$ & 1.000 \\
\hline
\end{tabular}

TABLE 6. Average irreversible entropy production, for R600. $k=v i s c$; mass; temp.

\begin{tabular}{|c|c|c|c|c|c|c|}
\hline \multicolumn{3}{|c|}{$t^{*}=105$} & \multicolumn{2}{|c|}{$t^{*}=120$} & \multicolumn{2}{|c|}{$t^{*}=135$} \\
\hline & $\left(\mathrm{W} \mathrm{m}^{-3} \mathrm{~K}^{-1}\right)$ & $g_{k}^{S G S} / g^{S G S}$ & $\left(\mathrm{~W} \mathrm{~m}^{-3} \mathrm{~K}^{-1}\right)$ & $g_{k}^{S G S} / g^{S G S}$ & $\left(\mathrm{~W} \mathrm{~m}^{-3} \mathrm{~K}^{-1}\right)$ & $g_{k}^{S G S} / g^{S G S}$ \\
\hline$g_{\text {visc }}^{S G S}$ & $2.17 \times 10^{4}$ & 0.065 & $1.88 \times 10^{4}$ & 0.061 & $1.96 \times 10^{4}$ & 0.068 \\
\hline $\begin{array}{l}\text { gemp } \\
g_{\text {temp }}^{S G S}\end{array}$ & $4.89 \times 10^{4}$ & 0.147 & $4.88 \times 10^{4}$ & 0.159 & $4.77 \times 10^{4}$ & 0.164 \\
\hline $\begin{array}{l}\text { gass } \\
g_{\text {mass }}\end{array}$ & $2.62 \times 10^{5}$ & 0.788 & $2.40 \times 10^{5}$ & 0.780 & $2.23 \times 10^{5}$ & 0.768 \\
\hline$g^{S G S}$ & $3.32 \times 10^{5}$ & 1.000 & $3.08 \times 10^{5}$ & 1.000 & $2.90 \times 10^{5}$ & 1.000 \\
\hline
\end{tabular}

TABLE 7. Average SGS irreversible entropy production, for R600. $k=$ visc; mass; temp.

\begin{tabular}{lcccccccc} 
& \multicolumn{2}{c}{$t^{*}=105$} & & \multicolumn{2}{c}{$t^{*}=120$} & & \multicolumn{2}{c}{$t^{*}=135$} \\
\cline { 2 - 3 } & $g_{k}^{S G S} / g$ & $g_{k}^{S G S} / g_{k}$ & & $g_{k}^{S G S} / g$ & $g_{k}^{S G S} / g_{k}$ & & $g_{k}^{S G S} / g$ & $g_{k}^{S G S} / g_{k}$ \\
$g_{\text {visc }}$ & 0.017 & 0.264 & & 0.014 & 0.243 & & 0.015 & 0.248 \\
$g_{\text {temp }}$ & 0.039 & 0.249 & & 0.036 & 0.220 & & 0.037 & 0.219 \\
$g_{\text {mass }}$ & 0.209 & 0.269 & & 0.179 & 0.231 & & 0.174 & 0.226 \\
$g$ & 0.265 & & & 0.230 & & & 0.226 &
\end{tabular}

TABLE 8. Small-scale contribution to the average dissipation and to the respective mode of dissipation for R600. $k=$ visc; mass; temp. The values of $g_{k}^{S G S} / g_{k}$ should not be calculated as the ratio of $g_{k}^{S G S} / g$ from this table to $g_{k} / g$ from table 6 , as round off errors yield incorrect values.

average dissipation is due to the small-scale action of the species mass flux, and the ratio $g^{S G S} / g$ slightly decreases as the transitional state is approached, reaching then about $23 \%$. This finding also contrasts with the situation for atmospheric-pressure mixing layers where the small-scale dissipation is of viscous origin. The contribution of the small scales to their respective average dissipation mode shown in table 8 is consistently highest for $g_{\text {mass }}$; the values of $g_{k}^{S G S} / g_{k}$ in table 8 should not be calculated as the ratio of $g_{k}^{S G S} / g$ from the same table to $g_{k} / g$ from table 6 , as round-off errors yield incorrect values. Remarkably, the small-scale contribution for all modes ranges between $22 \%$ and $27 \%$, a rather narrow range, and decreases as the transitional state is approached, this being attributable of the increasing destruction of small scales through dissipation.

To determine the location of the primary activity in the dissipation, homogeneous $\left(x_{1}, x_{3}\right)$-plane averages of $g_{\text {visc }}, g_{\text {temp }}, g_{\text {mass }}$ at $t^{*}=105,120$ and 135 were calculated; those at $t^{*}=135$ are illustrated in figures $9(a)$ (average) and 9(b) (RMS). At all three times, and consistent with the global analysis, the overwhelming activity is from $g_{\text {mass }}$, both for the average and the RMS. Prior to transition, the peaks of the average and RMS occur at the same location, which is at the boundary between the two fluids. As the transitional state is approached, the RMS peaked region narrows and exhibits sharper peaks, indicating an increased level of fluctuations. At transition, the 
DNS of a supercritical mixing layer
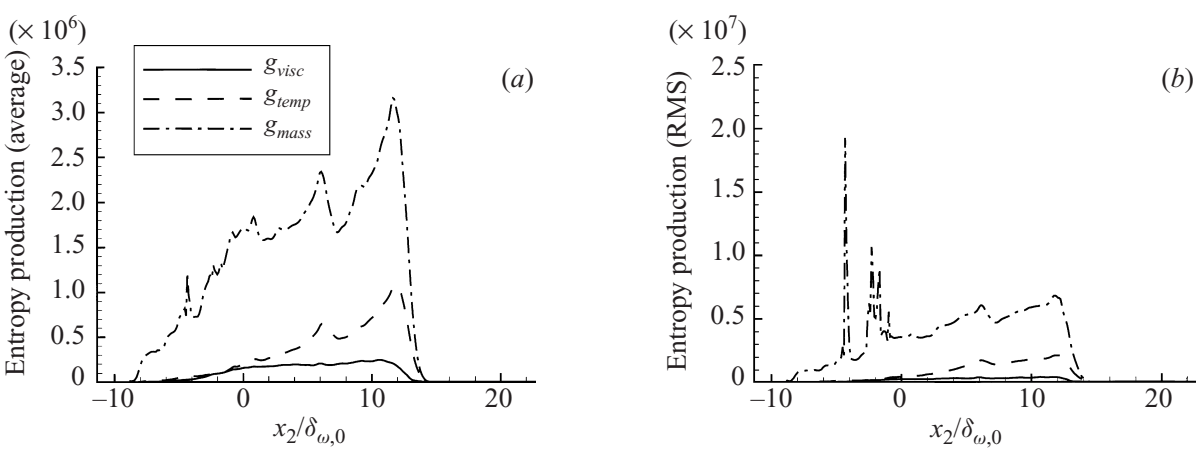

FIGURE 9. Contributions to dissipation for $R e_{0}=600$ at $t^{*}=135:(a)$ average, $(b)$ RMS.
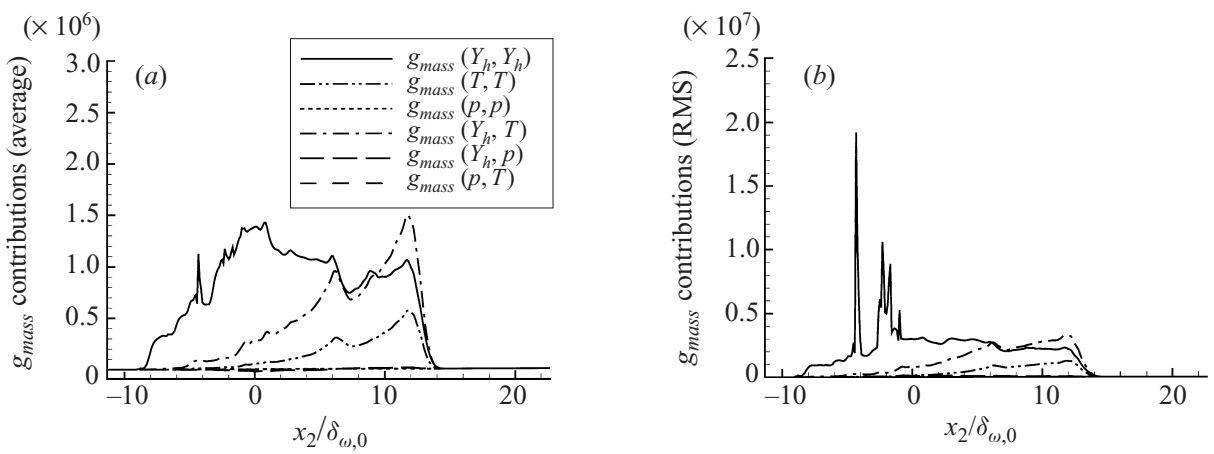

FIGURE 10. Contributions to the species mass flux dissipation, $g_{\text {mass }}$, for $R e_{0}=600$ at $t^{*}=135$ : (a) average, (b) RMS.

average (figure $9 a$ ) appears overwhelmingly located on the nitrogen side, where the mass fraction gradients are large, whereas the RMS has strong and sharp peaks on the heptane side of the layer, where most of the regions of high $|\nabla \rho|$ reside. These sharp peaks correspond to the pockets of strong dissipation detected on the heptane side of the layer in the contour plots. This indicates that although most of the average dissipation occurs on the nitrogen side of the layer, most of the fluctuations in the dissipation are associated with the region of high $|\nabla \rho|$, which they follow during the layer evolution. $g_{\text {temp }}$ is approximately one order of magnitude smaller than $g_{\text {mass }}$ and its maximum activity is on the nitrogen side of the layer where the temperature gradients are largest. The $g_{v i s c}$ contribution is negligible, being approximately two orders of magnitude smaller that of $g_{\text {mass }}$.

Because $g_{\text {mass }}$ is proportional to $j_{h j} j_{h j}$, and since $j_{h j}$ contains three terms (see (2.17) and (2.18)), it is intriguing to elucidate the contribution of each of the six terms to $g_{\text {mass }}$. Displayed in figure 10 are these various contributions, with the notation $g_{\text {mass }}\left(Y_{h}, Y_{h}\right) \propto\left(\alpha_{D} D\right)^{2} \nabla Y_{h} \cdot \nabla Y_{h}$, etc. Clearly, the Fick diffusion term contributes overwhelmingly to the average (figure $10 a$ ) on the heptane side of the layer, with significant contribution on the nitrogen side as well; at positions close to the nitrogen free stream, the cross-term $g_{\text {mass }}\left(Y_{h}, T\right) \propto \alpha_{D} D \alpha_{B K}$ dominates all other terms. The RMS (figure 10b) is even more strongly dominated by $g_{\text {mass }}\left(Y_{h}, Y_{h}\right)$ on the heptane side, and competes in magnitude on the nitrogen side with $g_{\text {mass }}\left(Y_{h}, T\right)$. The $g_{\text {mass }}(T, T)$ has a modest contribution to $g_{\text {mass }}$, and all other terms are negligible. These results highlight the importance of the molecular diffusion in determining the dissipation, 


\begin{tabular}{cccc}
\hline & $t^{*}=105$ & $t^{*}=120$ & $t^{*}=135$ \\
$g_{\text {visc }}$ & 34.7 & 33.8 & 33.2 \\
$g_{\text {temp }}$ & 42.8 & 39.4 & 40.4 \\
$g_{\text {mass }}$ & 49.2 & 45.9 & 46.5 \\
$g$ & 39.9 & 39.0 & 40.9 \\
TABLE 9. Backscatter of entropy dissipation modes for R600. The listed numbers are \% of points \\
in the domain where the SGS contribution of the quantity has a negative value.
\end{tabular}

and the significant role of the Soret effect in the modelling of supercritical transitional flows.

Similar calculations to those presented in table 8 conducted for the domainaveraged RMS (not displayed), instead of the average dissipation values, also show the dominance of the species flux contribution. Furthermore, the domain RMS of both $g_{\text {mass }}^{S G S} / g_{\text {mass }}$ and $g_{\text {mass }}^{S G S} / g$ are considerably larger than those of the average dissipation $\left(88 \%, 76 \%\right.$ and $58 \%$ for $g_{\text {mass }}^{S G S} / g_{\text {mass }}$, and $81 \%, 67 \%$ and $47 \%$ for $g_{\text {mass }}^{S G S} / g$ at $t^{*}=105$, 120 and 135), this being a manifestation of the important role of the subgrid scales in the RMS of the dissipation.

Of significance is the fact that equivalent tables (not shown) to tables 6 and 7 , now calculated for the RMS dissipation, show values that are always about one order of magnitude larger than the corresponding average values, indicating that the flow displays considerable backscatter. To ascertain the role of backscatter, listed in table 9 is the percentage of domain points where each of the dissipation modes, $g_{\text {visc }}, g_{\text {temp }}, g_{\text {mass }}$, and the total dissipation, $g$, are negative. For all dissipation modes there is a substantial number of points in the domain where backscatter occurs. The most widespread backscatter is manifested by the species mass flux dissipation mode, occurring over more than $49 \%$ of the domain at the earliest time and over $46 \%$ of the domain at transition. The regions of $g_{\text {temp }}$-generated backscatter are almost as widespread as those corresponding to $g_{\text {mass }}$ while the $g_{v i s c}$ activity is the most modest.

These results reveal that turbulence modelling in the context of supercritical mixing layers must concentrate on species mass flux rather than viscosity effects, as they govern all aspects of dissipation as well as backscatter. The regions of high density gradient magnitude are instrumental in providing the venue for the dissipation.

\subsection{PDF representation}

Probability density function (PDF) methods have received much attention in describing reacting flows (e.g. O’Brien 1980; Pope 1985, 1991; Frankel et al. 1993; Dopazo 1994) because they circumvent some of the need for turbulence modelling. One may distinguish two classes of PDF methods. In the first approach, the evolution equation for the PDF is solved. This method is potentially attractive primarily because of the inherent ease in treating reaction terms, which in this formulation do not need to be modelled. Although much progress has been made in this area (see Pope 1985, 1991; Dopazo 1994), there are still difficulties in the treatment of the diffusion terms, and the approach remains computationally expensive. The second PDF method is called the 'assumed PDF' approach and consists of assuming the mathematical form of the PDF to close the mean chemical source terms in the equations (e.g. Frankel et al. 1993). To adopt the assumed PDF approach, one needs to calculate a finite number of moments of the assumed PDF in order to construct it according to well-known methods in probability theory (Papoulis 1991). Generally, these moment equations 

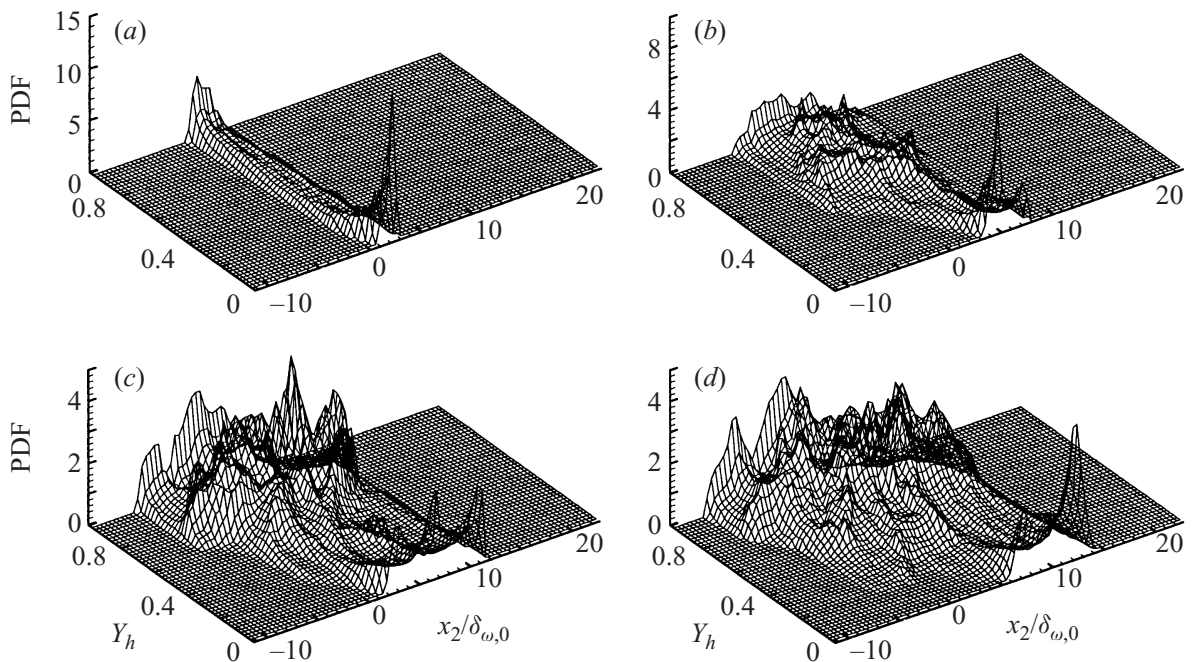

FIGURE 11. Planar PDF of heptane mass fraction for $R e_{0}=600$ at $(a) t^{*}=25$, (b) $t^{*}=75$, (c) $t^{*}=105,(d) t^{*}=135$.

are derived from the conservation equations, and the inherent unclosed terms thus formed are modelled according to the best known physics. The moment equations are then solved together with the other conservation equations. Two typical PDF forms used for atmospheric gaseous flows are the $\beta$ and the Gaussian densities, both of which are entirely defined from their first two moments. Their advantage is the accommodation of the description of completely segregated species with, for shear layers, initial peaks in the two streams ( $\beta$ density), and the asymptotic completely mixed state (Gaussian).

Here, we focus on the assumed PDF approach and address two questions. First, we are interested in exploring the possibility of using the assumed PDF approach for describing the evolution of the supercritical mixing layer from the segregated state to transition. The issue of the accurate calculation of the necessary moments (the solutions of modelled differential equations; see above) will not be addressed here. Instead, we will decouple the two separate issues of accurate moment calculation and assumed form of the PDF by adopting the values obtained by DNS in the construction of the PDFs. With the accurate moments, we will compare the assumed PDF with that extracted from the DNS to determine if the method would be appropriate for the present situation. This assumed PDF would represent the best attainable model since the moments are exact. Related to assumed PDF modelling, we also address the issue of whether the temperature and composition PDFs are correlated, since ascertaining this is of interest in the modelling of reaction rate terms through a joint PDF. The second question that we wish to address is that of the form of the PDF at the subgrid scale. Since LES have emerged as a powerful computational tool, it is of interest to inquire if PDF-based subgrid-scale modelling could be a useful approach.

\subsubsection{Assumed PDF representation}

Illustrations of the conserved scalar, here the species mass fraction, PDF are presented in figures $11(a)-11(d)$ for four different values of $t^{*}$ appropriately chosen to represent stations before the first pairing, after the first pairing, after the second pairing and at transition for R600; the results (not shown) are similar for R400 

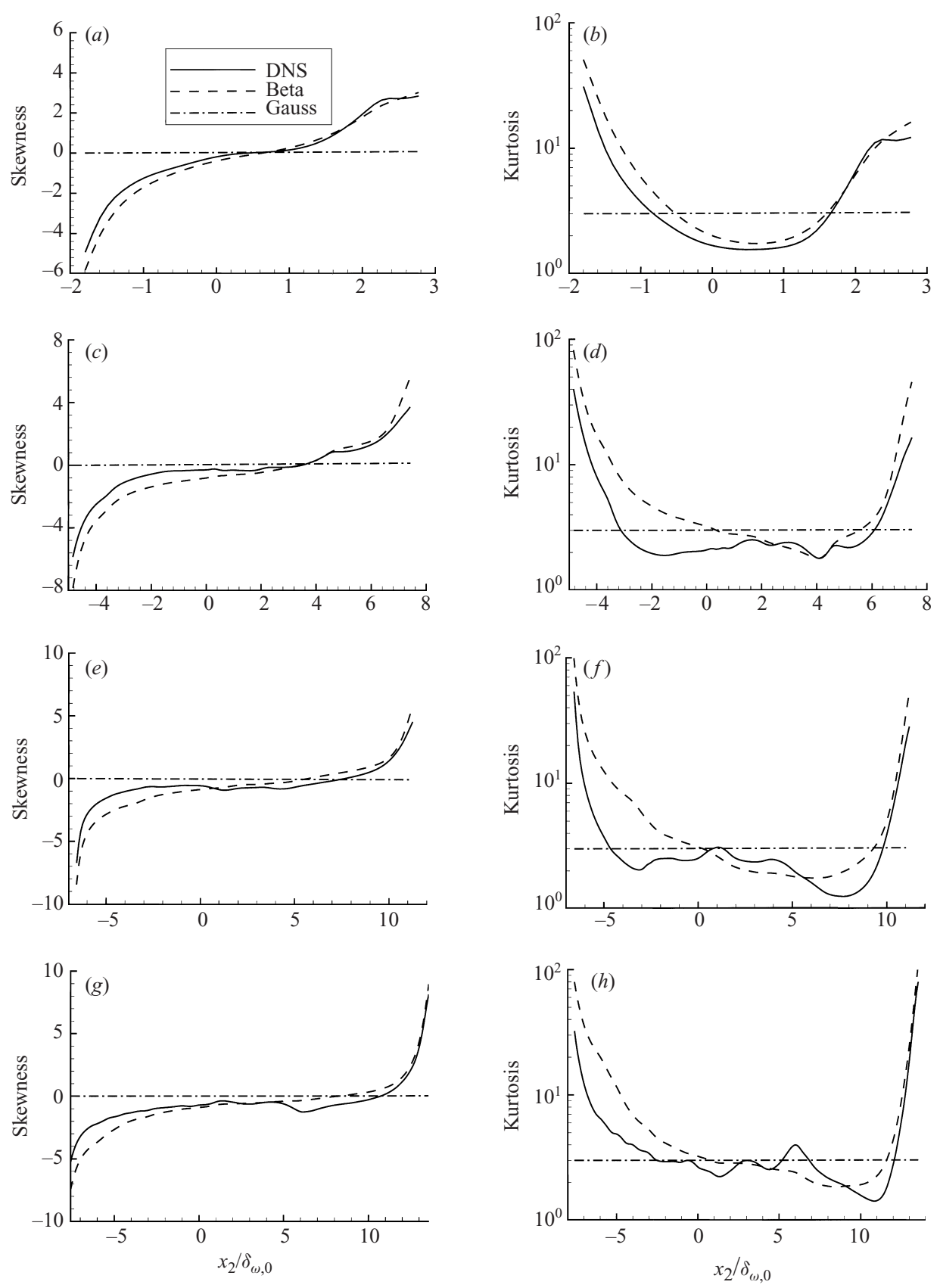

FIGURE 12. Planar PDF statistics of heptane mass fraction for $R e_{0}=600(a, b)$ at $t^{*}=25,(c, d)$ at $t^{*}=75,(e, f)$ at $t^{*}=105,(g, h)$ at $t^{*}=135$; skewness $(a, c, e, g)$ and kurtosis $(b, d, f, h)$.

and R500. In all these figures, the null and unity $Y_{h}$-planes were removed, to give a more discernible pictorial representation. Even at the earliest time, $t^{*}=25$, heptane has already penetrated into the upper stream; however, the general features are those of laminar mixing. Past the first pairing $\left(t^{*} \simeq 50\right)$, entrainment initiates the production of small scales and mixing, as depicted in figure 11(b) showing the PDF at $t^{*}=75$. Further pairing at $t^{*} \simeq 85$ induces additional entrainment with 

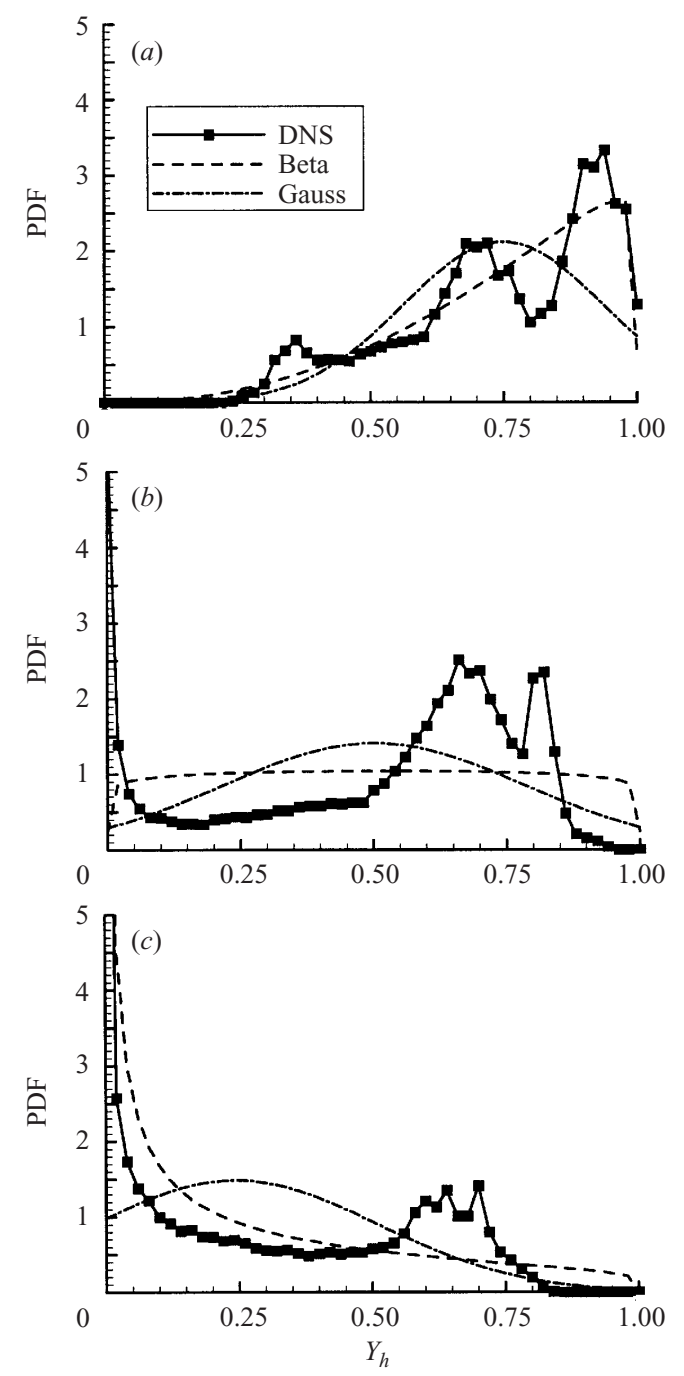

FIGURE 13. Planar PDF of heptane mass fraction compared to beta and Gaussian for $R e_{0}=600$ at $t^{*}=135$ in selected planes: $x_{2} / \delta_{\omega, 0}(a) 0.44,(b) 8.36,(c) 11.61$.

consequently increased mixing as evidenced in figure 11(c) showing the $t^{*}=105 \mathrm{PDF}$. Finally, at the transitional state, $t^{*}=135$, the very convoluted PDF of figure $11(d)$ exhibits the attributes of intense mixing.

To explore the possibility of these PDFs being represented by a Gaussian, the skewness, $\mathscr{M}_{3}=\left\langle(\phi-\langle\phi\rangle)^{3}\right\rangle / \sigma^{3}$, and kurtosis, $\mathscr{M}_{4}=\left\langle(\phi-\langle\phi\rangle)^{4}\right\rangle / \sigma^{4}$, where the standard deviation is $\sigma=\sqrt{\left\langle(\phi-\langle\phi\rangle)^{2}\right\rangle}$, were calculated at the four time stations for planes with $\left\langle Y_{h}\right\rangle$ between 0.005 and 0.995 and compared with the known respective values of 0 and 3 of the Gaussian. The results are displayed in figure 12(a) to 12( $h)$, where the skewness and the kurtosis of the $\beta$ density were plotted for comparison, with the $\beta$ statistics based on the exact (i.e. DNS calculated) mean and variance. Clearly, both the skewness and kurtosis of the DNS PDF approach the Gaussian values in the middle of the layer as the layer evolves towards the transitional state. Although the comparison is favourable in the middle of the layer, departures from 
the Gaussian values can be seen at the edge of the layer, indicating that perhaps the emulation of the DNS PDF by the Gaussian is not appropriate. On the other hand, the $\beta$-density skewness and kurtosis compare favourably with the DNS calculated values, indicating that possibly the $\beta$ PDF may be a good representation of the DNS PDF. To check this possibility, we calculated the planar PDF at $t^{*}=135$ and display it in figure 13. The three cross-stream locations at which the planar PDFs are illustrated are representative of the comparison between the DNS extracted PDF, the Gaussian and the $\beta$ density. It is evident that neither the Gaussian, nor the $\beta$ density can duplicate the humps of the exact PDF; this shows that approximate agreement of the moments does not necessarily imply agreement of the PDFs. Based on this limited comparison for three realizations, it appears that the much utilized PDF shapes for perfect gases and ideal mixtures may not be appropriate for real fluids and non-ideal mixtures.

A recent and comprehensive example of assumed PDF treatment is that of Baurle \& Girimaji (1999) who extended the typical utilization of this method, whereby the joint temperature-composition PDF is assumed to be the product of the marginal PDFs, by including cross-correlation effects between temperature and species partial densities; this study was conducted for atmospheric pressure. Baurle \& Girimaji (1999) correctly note that assuming the joint temperature-species PDF to be the product of the marginal PDFs implies that there is statistical independence between temperature and partial species densities. This assumption is questionable since in fact the EOS relates the temperature to the partial density. At high pressures where the flux matrix is enlarged to contain the additional influences of the mass fraction gradients on the temperature and vice versa, the temperature and partial-density PDFs might be even more correlated. To check whether the marginal PDFs are correlated, we calculated the correlation coefficient between the DNS-generated PDFs. It should be emphasized that because the present DNS does not include chemical reactions, which will necessarily induce a temperature and composition PDF correlation (see Baurle \& Girimaji 1999), our test of whether the two PDFs are correlated represents the most favourable outcome that could be expected for modelling the joint PDF as a product of the marginal PDFs. Displayed in figure 14(a) is the DNS correlation between $T$ and $\rho Y_{h}$ as a function of the cross-stream location. The temperature and partial density are clearly well and negatively correlated, indicating that the approximation of the joint PDF by the product of the marginal PDFs is inaccurate. The good correlation between the $T$ and $\rho Y_{h}$ PDFs is attributable to the form of the flux matrix, (2.6), showing the coupling between molar fluxes and temperature gradients and between the heat flux and mass fraction gradients; this coupling is negligible at atmospheric pressures where the flux matrix is uncoupled (see Harstad \& Bellan 1999). To emphasize the different characteristics of the joint PDF and the product of the marginal PDFs, plotted in figures $14(b)$ and 14(c) are contours of both of them. Notably, both the shape and the magnitude of the these two PDFs are different, consistent with the high correlation between $T$ and $\rho Y_{h}$.

\subsubsection{Subgrid-scale PDFs}

For the special case of top-hat filters, the filtered value is the volume average within the filtering volume, $\bar{\phi}=\left(\int_{V_{f}} \phi \mathrm{d} V\right) / V_{f}$, where $V_{f}$ is the filtering volume. In our calculations where each point has a filtering volume associated with it, when the flow field is filtered, each point within a given filtering volume may have a different value of $\bar{\phi}$. Thus, to emphasize that the PDFs are calculated within filtering volumes, we denote the filtered value (or volume average) for the filtering volume as $\bar{\phi}_{0}$; then, 

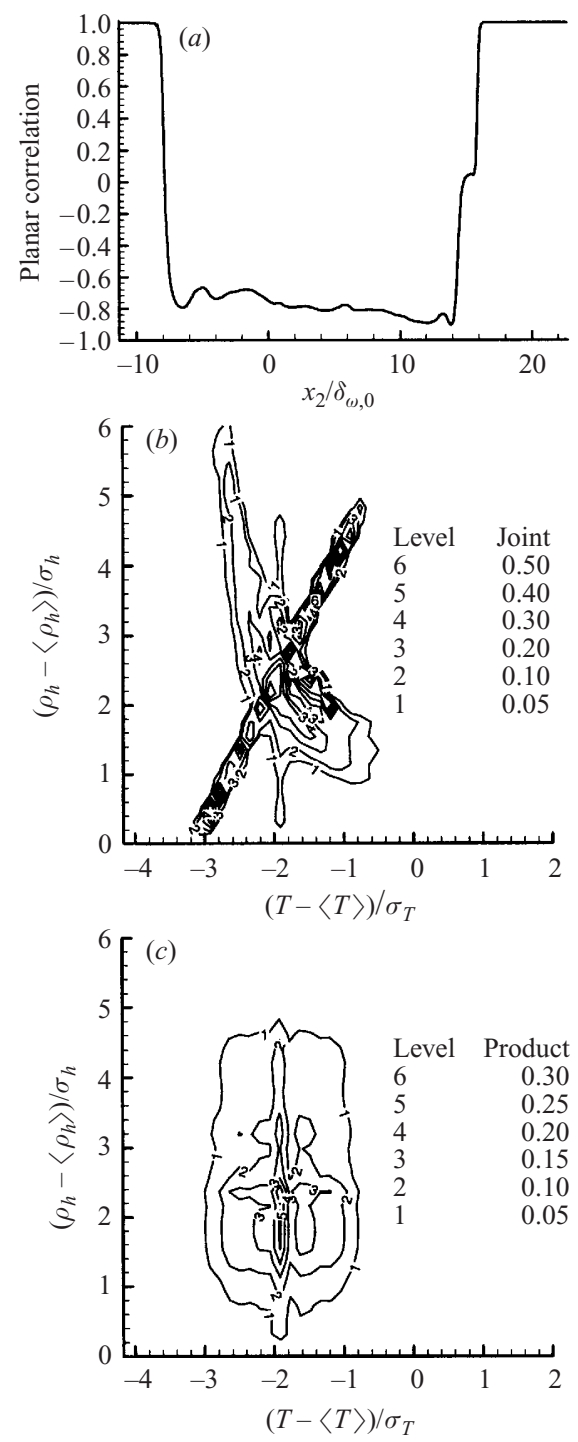

Figure 14. Correlation of heptane density and temperature for $R e_{0}=600$ at $t^{*}=135$ : (a) planar correlation, (b) joint PDF, $(c)$ product of marginal PDFs.

by definition each point within the filtering volume will have the same $\bar{\phi}_{0}$, which is equal to $\bar{\phi}$ only at the point with which this particular filtering volume is associated. According to Cook \& Riley (1994), the PDF within the filtering volume should be calculated relative to $\bar{\phi}_{0}$, with the subgrid standard deviation, $\sigma_{S G S}$, given by

$$
\sigma_{S G S}^{2}(\phi) \equiv \overline{\left(\phi-\bar{\phi}_{0}\right)^{2}}=\overline{\phi \phi-2 \phi \bar{\phi}_{0}+\bar{\phi}_{0} \bar{\phi}_{0}}=\overline{\phi^{2}}-\bar{\phi}^{2} .
$$

Therefore, any subgrid PDF calculated herein is centred at the Favre average mean $(\tilde{\phi}=\overline{\rho \phi} / \bar{\rho})$ in the filtering volume.

Pope (1991) promoted the concept of 'filtered density function', FDF, which is essentially the PDF of the variables at the subgrid scale. The FDF concept is potentially useful in reacting flows where the effects of the scalar fluctuations may be 


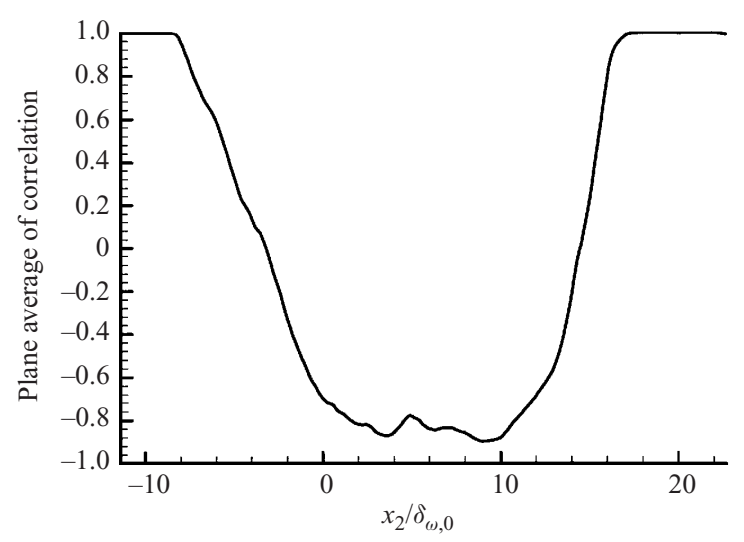

FIGURE 15. Correlations within filtering volumes between heptane density and temperature, plane averages for $R e_{0}=600$ at $t^{*}=135$.

modelled in a LES through the FDF. Colucci et al. (1998) have shown that a transport equation may be derived for the FDF in which the effect of the chemical reaction appears in closed form, but in which the effects of the scalar mixing and convection within the subgrid must be modelled. Here we wish to take an alternative direction to the approach of Colucci et al. (1998) by exploring the assumed PDF concept in the determination of the FDF, similar to the two PDF approaches (solution of the PDF equation versus assumed PDF method) at the larger scale. In this context, we inquire whether the subgrid reaction rate, which is a joint temperature-species PDF, may be assumed to be the product of the marginal PDFs. Illustrated in figure 15 is the correlation between $T$ and $\rho Y_{h}$ in the filtering volumes for R600 at $t^{*}=135$. Similar to the DNS PDF, the subgrid $T$ and $\rho Y_{h}$ (centred at the mean in each filtering volume) are well (negatively) correlated, indicating that the product of the marginal PDFs is not an accurate approximation of the subgrid reaction rate which is the joint PDF of the temperature and the partial species density.

To further explore the shape of the subgrid PDF, and the assumed PDF method for the passive scalar (here the species), displayed in figure 16(a) are the subgrid PDFs of all variables for R600 at $t^{*}=135$. Although the global shape of the PDFs is Gaussian, the numerous large peaks, especially those of $Y_{h}$ make it very uncertain that it can capture the specific physics of the situation. The origin of these peaks was investigated by performing another calculation where the PDFs were computed in the same manner as those in figure $15(a)$, except that the range of the variables was limited to be inside the layer. Since the layer has different widths according to the variable, $Y_{h}$ and $T$ were calculated in their own layer width, $u_{1}, u_{2}$ and $u_{3}$ were conservatively calculated in the narrowest layer width (that of $u_{1}$ ), and since the $p$ layer was not evident, the value adopted was that of the $T$ layer because of the strong coupling between these two variables through the EOS. The results are illustrated in figure $16(b)$. A comparison between figures $16(a)$ and $16(b)$ leads to the conclusion that the peaks in figure $16(a)$ are due to intermittency effects at the edge of the layer; clearly, the Gaussian profile cannot model these important intermittency effects. Moreover, examination of figure $16(b)$ reveals that even without the intermittency effects, the humps around the mean, especially large and asymmetric for $Y_{h}$, cannot be emulated by the Gaussian PDF. This comparison between the simulation derived PDFs and the Gaussian sheds doubt on the usefulness of the assumed FDF approach for supercritical flow subgrid-scale modelling. 

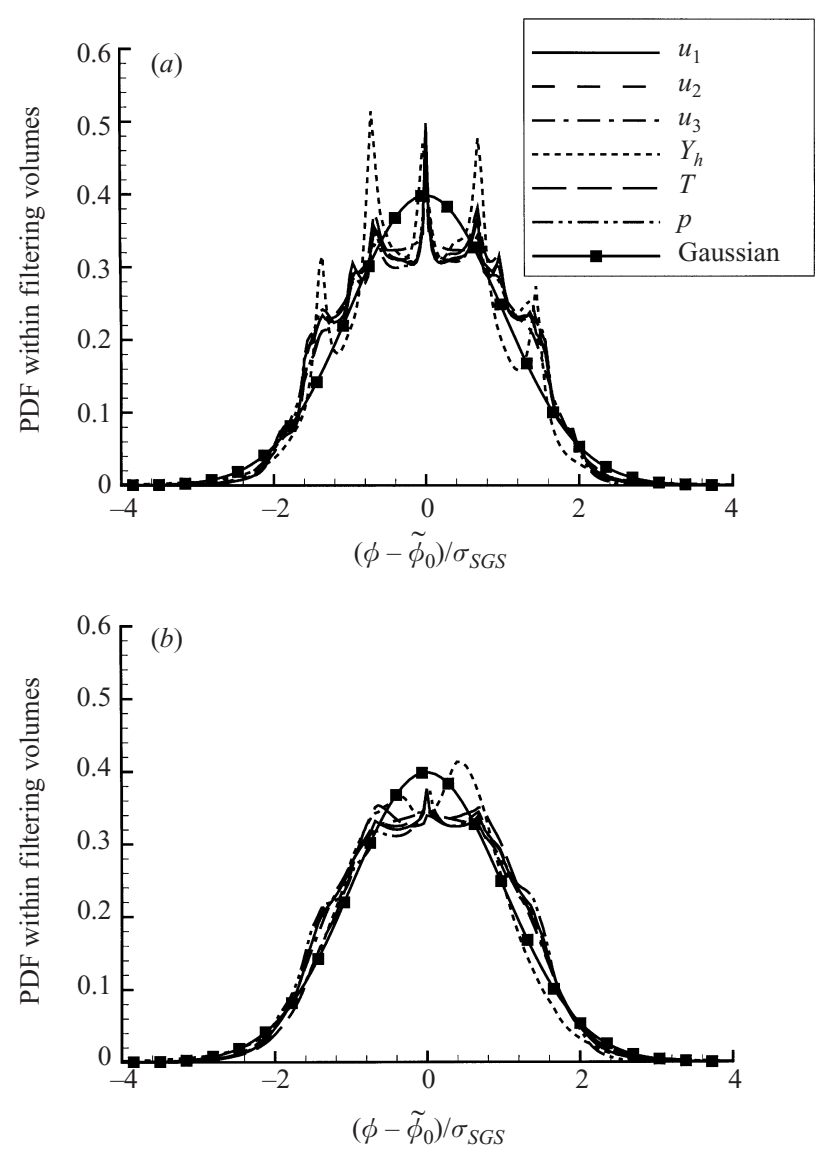

FIGURE 16. PDF within filtering volumes for $R e_{0}=600$ at $t^{*}=135$ of $u_{1}, u_{2}, u_{3}, Y_{h}, T$ and $p$ : $(a)$ within entire domain, $(b)$ within mixing layer.

\section{Summary and conclusions}

Specific aspects characterizing the behaviour of supercritical mixing layers have been elucidated through direct numerical simulations (DNS). The conservation equations contain the full transport matrix according to fluctuation-dissipation theory, incorporating thermal diffusion effects. The adopted value of the additional transport coefficient thus introduced is that determined in a previous study. The real gas equation of state used in conjunction with the dynamic conservation equations was also employed to calculate the mass diffusion factor which quantifies a mixture's departure from non-ideality. The Schmidt and Prandtl numbers were accurate over the thermodynamic range of the simulations, emulating values obtained from contour plots based on accurate transport properties. The boundary conditions were periodic in the streamwise and spanwise directions, and based on real-gas characteristic waves exiting the domain without reflection in the cross-stream direction.

Three simulations were conducted for a heptane (lower stream) and nitrogen (upper stream) configuration, having initial Reynolds numbers of 400, 500 and 600. All simulations were fully resolved, and for the largest Reynolds number the calculation encompassed in excess of $17 \times 10^{6}$ grid points. The three simulations were carried to mixing transition states obtained after two pairings of the initial four spanwise vortices 
present in the layer. The transitional state was identified by global manifestations such as rapid and sustained momentum thickness growth, high rate of positive spanwise vorticity evolution, increased enstrophy, large product thickness, and large momentum-thickness-based Reynolds number. Analysis of the vorticity and vorticity magnitude budgets at transition highlighted the dominance of the stretching and tilting effect in the production of spanwise vorticity, and of the viscous contribution in the vorticity magnitude, although at some cross-stream locations stretching and tilting exceeded viscous effects.

Visualizations of the spanwise vorticity at the transitional state revealed considerable local positive vorticity in both the braid and between-the-braid planes. Furthermore, the layer exhibited concentrated regions of high density gradient magnitude, which displayed a convoluted and distorted appearance; these regions are specific to the supercritical aspect of the layer. Given the commonality of distinctive optical features between temporal and spatial shear layers, the existing observation of such regions in supercritical jet experiments provides encouragement that the essence of the physics has been captured. Analysis of the fluid composition in these regions of high density gradient magnitude revealed that the predominant species is that in the lower, entrained stream with small amounts of the entraining fluid transported and dissolved into it. Moreover, considerations based on the value of the mass diffusion factor identified these regions as containing a highly non-ideal mixture, implying mixing difficulties at the molecular level resulting from the thermodynamic properties of the mixture. Visualizations of the compression factor displayed large departures from perfect gas behaviour in the lower heptane stream as well as in the mixing layer. Neither departures from perfect gas behaviour, nor deviations from mixture non-ideality have previously been identified in DNS of transitional mixing layers.

The novel thermodynamic characteristics of the layer prompted an investigation of the thermodynamic state of the mixture at transition through examination of departures from the critical locus in both the braid and between-the-braid planes. The fluid is supercritical everywhere, as the temperature is always larger than that of the local critical point. However, the pressure is above the local critical point outside the vortices and below the local critical point inside with the critical locus mapping the coherent vortices. The considerable reduction in the difference between the local values of the pressures and critical pressure inside the vortices is due to the change in fluid composition. These features are specific to the supercritical, transitional state of the layer.

To investigate the primary mechanisms responsible for dissipation both during the evolution of the layer and at transition, the irreversible entropy production was calculated and analysed. The three contributions to the dissipation arising from viscous stresses, species mass fluxes and heat fluxes were calculated and compared. Volume averages as well as RMS were computed at time stations after the second pairing, but prior to transition, and at transition. Moreover, by filtering the DNS solution and calculating the difference between the filtered and unfiltered variables, the contribution of the small scales (SGS) was evaluated. The overwhelming contribution both to the average and the RMS dissipation, as well as to the SGS dissipation, is due to species mass flux effects. The primary contribution to the species mass flux dissipation is due to Fick's diffusion term with an important participation of the product between the diffusion and the Soret terms. Furthermore, the species mass flux contribution to the dissipation has the most widespread backscatter. The viscous contribution is the smallest in all these important aspects of dissipation. 
Therefore, supercritical transitional mixing layers are fundamentally different from their atmospheric-pressure counterpart, which are dominated by viscous effects.

Since the transitional state is dominated by species mass flux effects, it is clear that turbulence models must predominantly focus on emulating the species mass fluxes and the Soret effects rather than the momentum flux (viscous stresses). Of significance is the observation that both the species mass flux and the Soret term will, in general, be species system specific. Therefore, ascertaining the species-dependent aspects of supercritical mixing layer behaviour will constitute one of our future priorities.

Assumed PDF modelling, of particular interest for reacting flow representations, was shown to have only limited potential. Neither the $\beta$ density nor the Gaussian PDF, both constructed using the DNS calculated moments, predict reliably the mixing process or the transitional state, respectively. The same result is found at the subgrid scale. Moreover, the temperature and partial density PDFs are well (negatively) correlated, indicating that the prospects of modelling the reaction rate, which is a joint temperature-species PDF, by the product of the marginal PDFs are poor. This good correlation was obtained both for the DNS-scale PDF and the SGS-scale PDF (the filtered density function of Pope 1991). Finally, it should be mentioned that the conclusions regarding the PDF representation are the result of studying only three realizations. Additional work will be necessary to obtain more definitive conclusions.

This research was conducted at the Jet Propulsion Laboratory (JPL), California Institute of Technology. Joint sponsorship was provided by the Air Force Office of Scientific Research with Dr Julian Tishkoff serving as contract monitor and by the Army Research Office with Dr David Mann as technical contract monitor, through interagency agreements with the National Space and Aeronautics Administration (NASA). Computational resources were provided by the supercomputing facility at JPL. The authors wish to thank Dr Mark Carpenter of NASA Langley Research Center and Dr Kenneth Harstad of JPL for helpful discussions.

\section{Appendix. Temperature fit}

Following Miller et al. (2001), the relationship between temperature and energy is taken as

$$
T=\left[\left(T_{L}\right)^{\theta}+\left(\frac{e-e_{L}}{e_{U}-e_{L}}\right)\left\{\left(T_{U}\right)^{\theta}-\left(T_{L}\right)^{\theta}\right\}\right]^{1 / \theta},
$$

where the subscripts $L$ and $U$ refer to the lower and upper limit of validity of the fit,

$$
\begin{gathered}
\theta=1.048+0.601 Y_{h}^{0.432}, \\
e_{L}=e_{1}+\left[\frac{\rho-\rho_{L}}{\rho_{U}-\rho_{L}}\right]\left(e_{2}-e_{1}\right), \\
e_{U}=e_{3}+\left[\frac{\rho-\rho_{L}}{\rho_{U}-\rho_{L}}\right]\left(e_{4}-e_{3}\right),
\end{gathered}
$$

and the upper and lower bounds are $\rho_{L}=6, \rho_{U}=286, T_{L}=500$ and $T_{U}=1100$ with the density in $\mathrm{kg} \mathrm{m}^{-3}$ and the temperature in $\mathrm{K}$ (this regime is larger than that of Miller et al. 2001). The limits of the density correspond to the pressure limits of 40 and $80 \mathrm{~atm}$ (see table 10 for pure species properties). Third-order polynomial fits were obtained for the four remaining internal energy functions $e_{1}\left(Y_{h} ; T_{L}, \rho_{L}\right), e_{2}\left(Y_{h} ; T_{L}, \rho_{U}\right)$, 


$\begin{array}{cclccc}\text { Species } & m\left(\mathrm{~kg} \mathrm{kmole}^{-1}\right) & T_{c}(\mathrm{~K}) & p_{c}(\mathrm{~atm}) & v_{c}\left(\mathrm{~m}^{3} \mathrm{kmole}^{-1}\right) & Z_{c} \\ \text { Nitrogen } & 28.013 & 126.26 & 33.55 & 0.0898 & 0.290 \\ \text { Heptane } & 100.205 & 540.3 & 27.04 & 0.432 & 0.263\end{array}$

TABlE 10. Pure species properties.

$\begin{array}{ccccc}\text { Energy }\left(\mathrm{J} \mathrm{kg}^{-1}\right) & E_{0} & E_{1} & E_{2} & E_{3} \\ e_{1}\left(Y_{h} ; T_{L}, \rho_{L}\right) & 3.6116 \times 10^{5} & 3.4529 \times 10^{5} & -1.3409 \times 10^{3} & 3.2521 \times 10^{0} \\ e_{2}\left(Y_{h} ; T_{L}, \rho_{U}\right) & 3.2562 \times 10^{5} & 2.8543 \times 10^{5} & -4.8335 \times 10^{4} & 2.5565 \times 10^{3} \\ e_{3}\left(Y_{h} ; T_{U}, \rho_{L}\right) & 8.6099 \times 10^{5} & 1.7846 \times 10^{6} & -1.4587 \times 10^{3} & 3.5423 \times 10^{0} \\ e_{4}\left(Y_{h} ; T_{U}, \rho_{U}\right) & 8.5152 \times 10^{5} & 1.7604 \times 10^{6} & -5.5374 \times 10^{4} & 3.0859 \times 10^{3}\end{array}$

TABLE 11. Polynomial curve fit coefficients.

$e_{3}\left(Y_{h} ; T_{U}, \rho_{L}\right)$ and $e_{4}\left(Y_{h} ; T_{U}, \rho_{U}\right)$ as follows

$$
e_{k}=E_{0}+E_{1} Y_{h}+E_{2} Y_{h}^{2}+E_{3} Y_{h}^{3}, \quad k=1,2,3,4,
$$

where the coefficients are provided in table 11. This particular fit achieves better than $1 \%$ maximum relative error when compared to the PR internal energy.

\section{REFERENCES}

AtsaVAPranee, P. \& GHaRib, M. 1997 Structures in stratified plane mixing layers and the effect of cross shear. J. Fluid Mech. 342, 53-86.

Baum. M., Poinsot, T. \& ThÉvenin, D. 1994 Accurate boundary conditions for multicomponent reactive flows. J. Comput. Phys. 116, 247-261.

BAurle, R. \& GRImaji, S. 1999 An assumed PDF turbulence-chemistry closure with temperaturecomposition correlations. Tech. Rep. 99-0928. AIAA.

Bellan, J. 2000 Supercritical (and subcritical) fluid behaviour and modelling: drops, streams, shear and mixing layers, jets and sprays. Prog. Energy Combust. Sci. 26, 329-366.

Bird, R., Stewart, W. \& Lightfoot, E. 1960 Transport Phenomena. John Wiley and Sons.

Brown, G. \& Roshko, A. 1974 On density effects and large structure in turbulent mixing layers. J. Fluid Mech. 64, 775-816.

Chapman, S. \& Cowling, T. 1970 The Mathematical Theory of Nonuniform Gases. Cambridge University Press.

Chenroudi, B., Talley, D. \& Coy, E. 1999 Initial growth rate and visual characteristics of a round jet into a sub- to supercritical environment of relevance to rocket, gas turbine and diesel engines. AIAA Paper 99-0206.

Chorin, A. 1994 Vorticity and Turbulence. Springer.

Colucci, P., Jaberi, F., Givi, P. \& Pope, S. 1998 Filtered density function for large eddy simulation of turbulent reacting flows. Phys. Fluids 10, 499-515.

Cook, A. \& Riley, J. 1994 A subgrid model for equilibrium chemistry in turbulent flows. Phys. Fluids 6, 2868-2870.

Cortesi, A., Yadigaroglu, G. \& BanerJee, S. 1998 Numerical investigation of the formation of three-dimensional structures in stably-stratified mixing layers. Phys. Fluids 10, 1449-1473.

Cortesi, A., Smith, B., Yadigaroglu, G. \& Banerjee, S. 1999 Numerical investigation of the entrainment and mixing processes in neutral and stably-stratified mixing layers. Phys. Fluids 11, $162-185$.

Dopazo, C. 1994 Recent developments in pdf methods. In Combustion Treatise. Turbulent Reacting Flows (ed. P. A. Libby \& F. A. Williams), pp. 375-474. Academic.

Frankel, S. H., Adumitroaie, V., Madnia, C. K. \& Givi, P. 1993 Large eddy simulation of 
turbulent reacting flows by assumed PDF methods. In Engineering Applications of Large Eddy Simulations (ed. S. A. Ragab \& U. Piomelli). ASME FED Vol. 162, pp. 81-101.

Hannoun, I. A., Fernando, H. J. S. \& List, E. J. 1988 Turbulence structure near a sharp density interface. J. Fluid Mech. 189, 189-209.

Harstad, K. \& Bellan, J. 1998 Isolated fluid oxygen drop behaviour in fluid hydrogen at rocket chamber pressures. Intl J. Heat Mass Transfer 41, 3537-3550.

Harstad, K. \& Bellan, J. 1999. The Lewis number under supercritical conditions. Intl J. Heat Mass Transfer 42, 961-970.

Harstad, K. \& Bellan, J. 2000 An all-pressure fluid-drop model applied to a binary mixture: heptane in nitrogen. Intl J. Multiphase Flow 26, 1675-1706.

Harstad, K., Miller, R. S. \& Bellan, J. 1997 Efficient high pressure state equations. AIChE J. 43, $1605-1610$.

Harstad, K. \& Bellan, J. 2001 Evaluation of commonly used assumptions for isolated and cluster heptane drops in nitrogen at all pressures. Combust. Flame 127, 1861-1879.

KeIzer, J. 1987 Statistical Thermodynamics of Nonequilibrium Processes. Springer.

Kennedy, C. A. \& Carpenter, M. H. 1994 Several new numerical methods for compressible shear-layer simulations. Appl. Numer. Maths 14, 397-433.

Kim, J., Moin, P. \& Moser, R. 1987 Turbulence statistics in fully developed channel flow at low Reynolds number. J. Fluid Mech. 177, 133-166.

Lin, S. J. \& CoRCos, G. M. 1984 The mixing layer: deterministic models of a turbulent flow. Part 3. The effect of plane strain on the dynamics of streamwise vortices. J. Fluid Mech. 141, 139-178.

Liu, S., Katz, J. \& Meneveau, C., 1999 Evolution and modelling of subgrid scales during rapid straining of turbulence. J. Fluid Mech. 387, 281-320.

Mayer, W., Schik, A., Schweitzer, C. \& Schaffler, M. 1996 Injection and mixing processes in high pressure LOX/GH2 rocket combustors. AIAA Paper 96-2620.

Mayer, W., Ivancic, B., Schik, A. \& Hornung, U. 1998 Propellant atomization in LOX/GH2 rocket combustors. AIAA Paper 98-3685.

Metcalfe, R. W., Orszag, S. A., Brachet, M. E., Menon, S. \& Riley, J. J. 1987 Secondary instability of a temporally growing mixing layer. J. Fluid Mech. 184, 207-243.

Miller, R. S. \& Bellan, J. 1999 Direct numerical simulation of a confined three-dimensional gas mixing layer with one evaporating hydrocarbon-droplet laden stream. J. Fluid Mech. 384, 293-338.

Miller, R. S. \& Bellan, J. 2000 Direct numerical simulation and subgrid analysis of a transitional droplet laden mixing layer. Phys. Fluids 12, 650-671.

Miller, R. S., Harstad, K. G. \& Bellan, J. 2001 Direct numerical simulations of supercritical fluid mixing layers applied to heptane-nitrogen. J. Fluid Mech. 436, 1-39.

Moser, R. D. \& Rogers, M. M. 1991 Mixing transition and the cascade to small scales in a plane mixing layer. Phys. Fluids A 3, 1128-1134.

Moser, R. D. \& Rogers, M. M. 1993 The three-dimensional evolution of a plane mixing layer: pairing and transition to turbulence. J. Fluid Mech. 247, 275-320.

Muller, S. M. \& Scheerer, D. 1991 A method to parallelize tridiagonal solvers. Parallel Computing 17, 181-188.

O'BRIEN, E. E. 1980 The probability density function (pdf) approach to reacting turbulent flows. In Turbulent Reacting Flows (ed. P. A. Libby \& F. A. Williams). Topics in Applied Physics, Vol. 44, pp. 185-216. Springer.

OKong'o, N. \& Bellan, J. 2000 Entropy production of emerging turbulent scales in a temporal supercritical n-heptane/nitrogen three-dimensional mixing layer. Proc. Combust. Inst. 28, 497 504.

Okong'o, N. \& Bellan, J. 2002 Consistent boundary conditions for multicomponent real gas mixtures based on characteristic waves. J. Comput. Phys. 176, 330-344.

OsCHWALD, M. \& SCHIK, A. 1999 Supercritical nitrogen free jet investigated by spontaneous Raman scattering. Exps. Fluids 27, 497-506.

Papoulis, A. 1991 Probability, Random Variables, and Stochastic Processes, 3rd edn. McGraw-Hill.

PENG, D.-Y. \& Robinson, D. B. 1977 A rigorous method for predicting the critical properties of multicomponent systems from an equation of state. AIChE J. 23, 137-144.

Poinsot, T. J. \& Lele, S. K. 1992 Boundary conditions for direct numerical simulations of compressible viscous flows. J. Comput. Phys. 101, 104-129. 
Pope, S. B. 1985 PDF methods for turbulent reactive flows. Prog. Energy Combust. Sci. 11, 119-192. Pope, S. B. 1991 Computations of turbulent combustion: progress and challenges. Proc. Combust. Inst. 23, 591-612.

Sarman, S. \& Evans, D. J. 1992 Heat flux and mass diffusion in binary Lennard-Jones mixtures. Phys. Rev. A 45, 2370-2379.

Slessor, M. D., Bond, C. L. \& Dimotakis, P. E. 1998 Turbulent shear-layer mixing at high Reynolds numbers: effect of inflow conditions. J. Fluid Mech. 376, 115-138. 